\title{
Single-cell multi-region dissection of brain vasculature in Alzheimer's Disease
}

Na Sun ${ }^{1,2}$, Leyla Anne Akay ${ }^{1,2,3,4}$, Mitchell H. Murdock ${ }^{3,4}$, Yongjin Park ${ }^{1,2,5,6}$, Adele Bubnys ${ }^{3,4}$, Kyriaki Galani $^{1,2}$, Hansruedi Mathys ${ }^{3,4,7}$, Xueqiao Jiang ${ }^{3,4}$, Ayesha P. Ng ${ }^{3,4}$, David A. Bennett ${ }^{8}$, Li-Huei Tsai ${ }^{2,3,4, *}$, Manolis Kellis ${ }^{1,2, *}$

1, MIT Computer Science and Artificial Intelligence Laboratory, Cambridge, MA, USA. 2, Broad Institute of MIT and Harvard, Cambridge, MA, USA. 3, Picower Institute for Learning and Memory, Massachusetts Institute of Technology, Cambridge, MA, USA. 4, Department of Brain and Cognitive Sciences, Massachusetts Institute of Technology, Cambridge, MA, USA. 5, Department of Pathology and Laboratory Medicine, Department of Statistics, University of British Columbia, Vancouver, BC, Canada. 6, Department of Molecular Oncology, BC Cancer, Vancouver, BC, Canada. 7, Department of Neurobiology, University of Pittsburgh School of Medicine, Pittsburgh, PA 15261, USA. 8, Rush Alzheimer's Disease Center, Rush University Medical Center, Chicago, IL, USA. * These authors jointly supervised this work: Manolis Kellis, Li-Huei Tsai. Correspondence to: manoli@mit.edu; Ihtsai@mit.edu

\section{Abstract}

Cerebrovascular breakdown occurs early in Alzheimer's Disease (AD), but its cell-type-specific molecular basis remains uncharacterized. Here, we characterize single-cell transcriptomic differences in human cerebrovasculature across $220 \mathrm{AD}$ and 208 control individuals and across 6 brain regions. We annotate 22,514 cerebrovascular cells in 11 subtypes of endothelial, pericyte, smooth muscle, perivascular fibroblast, and ependymal cells, and how they differ in abundance and gene expression between brain regions. We identify 2,676 AD-differential genes, including lower expression of PDGFRB in pericytes, and ABCB1 and ATP10A in endothelial cells. These AD-differential genes reveal common upstream regulators, including MECOM, EP300, and KLF4, whose targeting may help restore vasculature function. We find coordinated vasculature-glial-neuronal co-expressed gene modules supported by ligand-receptor pairs, involved in axon growth/degeneration and neurogenesis, suggesting mechanistic mediators of neurovascular unit dysregulation in AD. Integration with AD genetics reveals 125 AD-differential genes directly linked to AD-associated genetic variants (through vasculature-specific eQTLs, Hi-C, and correlation-based evidence), 559 targeted by AD-associated regulators, and 661 targeted by AD-associated ligand-receptor signaling. Lastly, we show that APOE4-genotype associated differences are significantly enriched among AD-associated genes in capillary and venule endothelial cells, and subsets of pericytes and fibroblasts, which underlie the vascular dysregulation in APOE4associated cognitive decline. Overall, our multi-region molecular atlas of differential human cerebrovasculature genes and pathways in $A D$ can help guide early-stage $A D$ therapeutics.

\section{Introduction}

The blood-brain-barrier (BBB) separates brain parenchyma from peripheral blood ${ }^{1}$. Multiple cell types, including endothelial cells, pericytes, and astrocytes, form the tight structure of BBB. This barrier prevents the entrance of pathogens and toxic substrates into the brain, but also presents a major challenge in drug delivery to the brain ${ }^{2}$. Vascular cells also supply neuronal and glial cells with nutrients and remove waste products, dynamically responding to the changing activity-dependent local energy 
demands stemming from the brain's large energetic needs and the lack of local energy storage, through tight cellular interactions and communication between neuronal, astrocyte, and vascular cells forming the neurovascular unit that senses neuronal activity, controls blood flow, and maintains BBB integrity.

BBB breakdown may be an early feature of Alzheimer's disease (AD), preceding dementia and neurodegeneration, suggesting a critical role of neurovascular unit dysfunction in the progression of $A D^{3,4}$. This leads to the entrance of toxic molecules, pathogens, and cells from peripheral blood to the central nervous system (CNS), triggering inflammatory and immune responses ${ }^{5,6}$. Impaired BBB function is associated with multiple neurodegenerative diseases in addition to $A D$, including multiple sclerosis (MS) and Parkinson's disease (PD) in human and mouse model studies ${ }^{6-8}$. In fact, the vascular hypothesis of $A D$ proposes brain vascular damage as the initial event catalyzing BBB dysfunction, precipitating brain dysfunction and cognitive decline ${ }^{9,10}$. However, whether AD risk genes regulate vascular function remains poorly understood systematically.

Brain vascular cells have evaded unbiased characterization due to technical challenges in their isolation, and the cellular complexity of the vascular arbor. The combination of single nucleus RNA sequencing (snRNA-seq) technologies and vessel enrichment protocols has provided an atlas of brain vasculature cell types in human and mouse ${ }^{11-13}$. However, cell sorting and enrichment protocols can introduce technical biases in cell type composition, and result in inaccurate spurious cell compositional inferences, especially if the marker genes used for sorting and enrichment change expression in the context of disease, leading to differences in capture efficiency. Thus, molecular characterization of human cerebrovascular cell types using sorting-free and enrichment-free methods is still needed to understand the cellular basis of the neurovascular unit. Moreover, different brain regions have been shown to possess cellular, morphological and functional differences in vasculature ${ }^{14}$. Understanding this molecular heterogeneity can provide insights into the unique vulnerabilities of different brain regions to disease.

Here, we address these challenges and report a single-cell characterization of the human cerebrovasculature in post-mortem samples from 6 brain regions across 220 AD and 208 age-matched control individuals. We use in-silico sorting to capture 22,514 cerebrovascular cells in 11 subtypes, including endothelial, pericytes, smooth muscle cells, perivascular fibroblasts, and ependymal cells. Comparing between brain regions, we find substantial differences in cell type proportion and in gene expression patterns between brain regions, highlighting the regional heterogeneity of the BBB for each cell type. Comparing between AD and non-AD individuals, we find 2,676 cell-type-specific differentially-expressed genes, and we predict their upstream regulators, whose targeting may help restore vasculature function. We also find ligand-receptor-supported coordinated co-expressed gene modules be- 
neurogenesis, suggesting mechanistic mediators of neurovascular unit dysregulation in AD. Integration with $A D$ genetics reveals $125 \mathrm{AD}$-differential genes directly linked to $A D$-associated genetic variants (through vasculature-specific eQTLs, Hi-C, and correlation-based evidence), 559 targeted by ADassociated regulators, and 661 targeted by $A D$-associated ligand-receptor signaling. Lastly, we show that APOE4-genotype associated differences are significantly enriched among AD-associated genes in capillary and venule endothelial cells, and subsets of pericytes and fibroblasts, which underlie the vascular dysregulation in APOE4-associated cognitive decline.

\section{Results}

\section{Brain vasculature characterization across six brain regions}

To characterize human cerebrovascular cells and their transcriptomic differences in $A D$ at single-cell resolution, we profiled and analyzed the transcriptome of 22,514 single nuclei from 725 post mortem brain samples of 220 AD and 208 control individuals (contributing 10,272 and 12,242 nuclei respectively, Supplementary Table 1) across 6 brain regions, selected using in silico sorting using both known marker genes and de novo clustering (Methods) ${ }^{12}$. We profiled the prefrontal cortex for 409 individuals, and five other regions for a subset of 48 individuals, including mid-temporal cortex, angular gyrus, entorhinal cortex, thalamus and hippocampus, as well as hippocampus for an additional 19 individuals (Supplementary Table 1).

We annotated 11 vascular cell types, including three types of endothelial cells (marked by FLT1, CLDN5), two types of pericytes (marked by RGS5, PDGFRB), two types of smooth muscle cells (SMCs) (marked by ACTA2), three types of fibroblasts (marked by COL3A1), and ependymal cells (marked by TTR) (Fig. 1a-b), using expression of canonical markers ${ }^{11,12}$ (Fig. 1c,d). Consistent with two recent studies ${ }^{12,13}$, we found distinct transcriptomic signatures of arterial, capillary, and venule endothelial cells, as well as both arterial and venule SMCs, suggesting functional specialization for different types of vessels. The inclusion of ependymal cells by our marker-based in silico cell sorting highlights the transcriptional commonalities of cerebrospinal fluid (CSF) and BBB barriers, as ependymal cells are not part of cerebrovasculature, but instead form a thin membrane that lines the ventricles of the brain and the central column of the spinal cord where CSF is produced.

We used transcriptional differences between cell types to highlight key biological functions for each vascular cell type in the brain through pathway-level enrichments of the most highly expressed genes of each cell type (Extended Data Fig. 1a-b, Supplementary Table 2-3, Methods). For endothelial cells, the highly expressed genes were most enriched in vascular endothelial growth factor receptor signaling pathway, regulation of cell migration, cytokine response, and cell junction assembly. For pericytes, the highly expressed genes were significantly enriched in pathways including the regulation of angiogenesis, extracellular matrix organization, endothelial cell migration, and cellular sodium ion ho- 
meostasis. For SMCs, the highly expressed genes were enriched in smooth muscle contraction, extracellular matrix organization, and regulation of cell junctions. For fibroblasts, the highly expressed genes were enriched in extracellular matrix organization, collagen organization, and regulation of cell migration. In ependymal cells, the highly expressed genes were enriched in cilium assembly, intraciliary transport and ciliary basal body-plasma membrane docking. These results highlight differential functional contributions of brain vascular cells to the maintenance of BBB, regulation of cerebral blood flow, and response to injury.

To gain insights into the gene regulatory programs responsible for establishing cerebrovascular-cell diversity, we next predicted upstream regulators whose activities are associated with the statues of molecular function and cell identity. We performed regulator enrichment analysis for marker genes of each cell type and found that the major cell types tended to share upstream regulators, but still show subtype specificity (Fig. 1e). Among the top regulators of cell identity, we found that some regulators show high enrichment in endothelial cells, including CTNNB1, which is associated with maintenance of BBB integrity through endothelial $\beta$-Catenin signaling ${ }^{15}$; LMO2, associated with endothelial cell migration in developmental and postnatal angiogenesis ${ }^{16}$; and ETS1, associated with endothelial cell survival in angiogenesis ${ }^{17}$. For pericytes, we found enrichment of $\mathrm{BACH} 1$, consistent with its transcriptional regulation of pro-angiogenic activity via modulating the expression of angiopoietin-1 ${ }^{18}$. We also found that the AD associated gene YAP1 is a common regulator in VSMC, fibroblast and ependymal cells, consistent with its roles in VSMC phenotypic switch ${ }^{19}$, fibroblast differentiation ${ }^{20}$, and ependymal integrity ${ }^{21}$.

We next evaluated whether vascular cells show differences in abundance across brain regions and phenotypic variables. Fibroblasts were enriched in entorhinal cortex, hippocampus and thalamus (Fig. 1f-g), consistent with increased vascular fibrosis and calcification of hippocampus and entorhinal cortex associated with aging ${ }^{22-25}$, and basement membrane and extracellular matrix regional differences $^{26}$, likely stemming from fibroblast-secreted collagen and other proteins (Fig. 1h). Ependymal cells were largely captured from the hippocampus and thalamus (Fig. 1f-g), consistent with these brain regions' proximity to CSF ventricles ${ }^{27}$. Hippocampus, thalamus, and entorhinal cortex also showed fewer pericytes and capillary endothelial cells, consistent with their paucity of small vessels, as those are locations where large vessels enter the brain ${ }^{28}$. By contrast, vascular cell fractions did not differ by sex, AD pathology, age, or post-mortem interval (PMI) (Wilcoxon Rank Sum test, $p$-value < 0.05, Extended Data Fig. 1c-j).

We found that vasculature cells showed extensive gene expression differences between brain regions, with many region-specific pathway enrichments, highlighting the regional heterogeneity of the BBB, and importance of single-cell multi-region characterization of the cerebrovasculature (Fig. 1h). We found 1,636 differentially-expressed genes between brain regions (brDEGs) (Supplementary Tables 
130

131

132

133

134

135

136

137

138

139

140

141

142

143

144

145

146

147

148

149

150

151

152

153

154

155

156

157

158

159

160

161

162

163

4), including: 230 endothelial brDEGs, enriched in molecule transport, regulation of cell migration and junction assembly; 491 pericyte brDEGs, enriched in calcium ion response in prefrontal cortex, and arterial blood pressure, cell junction assembly and response to low-density lipoprotein stimulus in hippocampus; 529 fibroblast brDEGs showing region-specific functional enrichments including extracellular matrix organization, exocytosis, immune response, ion homeostasis, and cell migration regulation; and 491 SMC brDEGs enriched in cell communication in prefrontal cortex, myelination in thalamus, muscle relaxation in hippocampus, and Notch signaling regulation in mid-temporal cortex. Several pathways were commonly enriched across multiple brain regions, including apoptosis regulation, cytokine response, and myeloid cell differentiation (Extended Data Fig. 1k, Supplementary Tables 5).

\section{Cell-type-specific brain vasculature differences in AD}

To investigate the vascular gene expression association with AD pathology, we identified and analyzed 2,676 differentially expressed genes (adDEGs) between AD and control individuals across all cell types (306 on average for each cell type), which is significantly more than expected using permutation analysis (t-test p-value=0.007, Extended Data Fig. 2a, Methods). Of these, 2,142 were unique to only one cell type, 185 (23 genes on average in 8 comparisons) were shared between subtypes of the same cell type (for example, 88 between cEndo and vEndo, and 50 between Per1 and Per2), and 349 (3.8 genes on average in 92 comparisons) were between cell types, highlighting the cell-typespecificity of adDEGs (Fig. 2a-b, Supplementary Table 6). This suggests that specialized functions of distinct cell types play unique roles to maintain brain homeostasis and may be dysregulated in $A D$, while the shared effect across cell types may represent a convergent response to AD. Notably, we found that capillary endothelial cells harbored the highest number of adDEGs, which we recapitulated through a downsampling analysis (Extended Data Fig. 2b), suggesting the importance of transcriptional differences in capillary endothelial functions associated with AD pathology (Fig. 2a).

Among the top adDEGs, we observed cell junction and adhesion associated genes including APOD, PECAM1, and COLEC12; transporters including SLC38A2, SLC2A1 and SLC6A1; and sterol-import associated genes including RORA, PRKAA2, and PPARG (Fig. 2c), indicating that these fundamental functions of vascular cell types may be dysregulated in AD. As expected, we detected lower expression of PDGFRB in pericytes of AD samples (Fig. 2c), alluding to pericyte injury and dysfunction of $B B B$ integrity in $A D^{4,29}$. We also found that $A B C B 1$, encoding P-glycoprotein, was significantly lower in capillary endothelial cells (Fig. 2c), consistent with observations that individuals with early AD develop widespread reductions in P-glycoprotein BBB function in multiple brain regions ${ }^{30,31}$.

Gene Ontology enrichment analysis of adDEGs showed that multiple broad functional pathways (e.g. immune response, insulin signaling, and vasculogenesis) were shared across cell types, and multiple specific pathways were more cell type specific (Fig. 2d-g, Extended Data Fig. 2c, Supplementary 
164

165

166

167

168

169

170

171

172

173

174

175

176

177

178

179

180

181

182

183

184

185

186

187

188

189

190

191

192

193

194

195

196

197

198

199

Table 7, Methods). Broad terms included: immune response (cytokine, IL-17 signaling, and inflammatory response) enriched in fibroblast, endothelial cell, and pericyte adDEGs; insulin response enriched in cEndo, pericyte, fibroblast, SMC and ependymal adDEGs, suggesting a potential functional link between altered glucose homeostasis, insulin signaling, and AD pathology across multiple vascular cells $^{32,33}$; vasculogenesis, endothelial cell migration and proliferation, cell-matrix adhesion, and ion transport enriched in cEndo and pericyte adDEGs (Fig. 2d-f). Specific terms included: in pericyte adDEGs with lower expression in AD, synaptic transmission (Fig. $\mathbf{2 g}$ ), consistent with pericyte loss of neuronal signal sensing in $\mathrm{AD}^{34}$, and cytoskeleton remodeling and contraction (e.g., ATP1A2, ANK2, DMD), consistent with impaired blood flow control and perturbed neurovascular coupling in pericytes in $A D^{35}$; in cEndo, Notch signaling regulation and endothelium development, consistent with the potential role of endothelial Notch signaling and dysfunction of angiogenesis in $A D^{36,37}$; in aSMC with lower expression in $A D$, cellular response to amyloid- $\beta$, consistent with findings showing that amyloid- $\beta$ deposits contribute to vascular alteration in $A D^{38}$.

Notably, our analysis specifically revealed that insulin signaling genes were AD-differential across multiple cell types (cEndo, pericyte, fibroblast, SMC and ependymal, Extended Data Fig. 2c) and in multiple brain regions. Insulin's cognate receptor INSR is widely expressed throughout the mammalian brain, including the hippocampus ${ }^{39}$, cortex ${ }^{40}$, olfactory bulb ${ }^{41}$ and hypothalamus ${ }^{42}$, and research has found aberrant insulin signaling in AD and related dementias ${ }^{43-45}$. To validate our snRNA-based observation that the insulin receptor INSR was highly expressed in endothelial cells from persons with $A D$, we performed in situ hybridization (Methods), and found CD31+ vascular segments harbored a higher density of INSR1 transcripts in those with AD. We also quantified the distribution of INSR ${ }^{+}$punctae per $\mathrm{CD} 31^{+}$segment, and found a subset of $\mathrm{CD} 31^{+}$endothelial cells from AD brains possessing higher INSR transcripts (Fig. 2h-i, Extended Data Fig. 2d-e). These results validate our observation that INSR is highly expressed in endothelial cells in $A D$, and suggest that subsets of endothelial cells may express differential levels of the insulin receptor, potentially rendering cells more or less sensitive to insulin signaling. The degree of heterogeneity of insulin receptor expression in vascular cells, and potential consequences to the neurovascular unit, remain an open question. As sensitivity to insulin signaling is an evolutionarily-conserved mediator of longevity ${ }^{46}$, our results provide further evidence that disruption to insulin is a pathological feature of $A D$.

We also observed that APOD, encoding secreted glycoprotein Apolipoprotein D, a component of highdensity lipoprotein $(H D L)$, is higher in AD samples in mural cells (particularly pericytes, $P<3.9 e-12$, Fig. 2c), in accordance with the previously-reported upregulation of $A P O D$ in $A D^{47}$. To validate its higher expression in AD pericytes, we quantified APOD transcript abundance in GRM8-labeled cells (a previously-validated marker for pericytes ${ }^{12}$ ) using in situ hybridization and observed that APOD expression was indeed higher in $\mathrm{GRM}^{+}$pericytes in AD individuals (Fig. 2j-k, Extended Data Fig. 2f) we did not use PDGFRB as the pericyte marker as it is lowly expressed in AD. Given the function of 
APOD in response to stress and injury in $\mathrm{CNS}^{48}$, the higher expression of APOD in AD pericytes suggests that mural cells are actively responding to microenvironmental changes during AD. The functional consequences of APOD's pericyte higher expression, and potential impacts on lipid transport within the neurovascular unit, will form the basis of future studies.

Our results show no significant change in vascular cell type proportion between non-AD and AD individuals (Extended Data Fig. 2g), and in fact a modest but non-significant high in the median number of capillary endothelial cells and pericytes in AD individuals. These results are consistent with an observed higher proportion of endothelial cells in $A D$ in two recent studies ${ }^{49,50}$ that also did not rely on any cell-type- or vessel-enrichment protocols. However, we found that several our adDEGs that showed lower expression in AD were cell type marker genes, including PDGFRB (pericytes), ABCB1, ATP10A, PTPRB and TEK (capillary endothelial cells), suggesting potential loss of vascular cell type integrity in AD. Such loss of vascular integrity, and lower expression of cell-type-specific markers, could result in a seeming decrease in vascular cell proportions in studies that rely on enrichment protocols.

\section{Upstream regulators of adDEGs}

215 To gain insights into the transcriptional regulatory mechanisms, we inferred the upstream regulators of adDEGs, including transcription factors, co-factors, and epigenetic enzymes (Methods, Supplementary Table 8). We identified 118 upstream regulators of highly expressed adDEGs (60 cell-type-specific, 58 shared by at least two cell types) (Fig. 3a) and 81 regulators of lowly expressed adDEGs (41 cell-type-specific, 40 cell-type-shared) (Fig. 3b), of which 66 targeted both highly and lowly expressed adDEGs across cell types in AD. Of these 133 regulators, 17 were themselves significant adDEGs corresponding to the differential direction of their targets.

We next grouped these regulators into co-regulatory modules for each cell type (Fig. 3c, Extended

Data Fig. 3), when regulators showed significant sharing of target genes. Focusing on capillary endothelial cells (cEndo), which showed the largest number of adDEG and upstream regulators, we identified seven regulatory modules (Fig. 3d) encompassing 38 regulators, and 11 regulators outside modules. Although our modules were discovered solely based on their shared target genes, most were additionally supported by independent experimental evidence of interactions ${ }^{51}$ (Fig. 3e); for example, Module M1 regulators showed a nearly-complete clique of pairwise interactions, each supported by multiple lines of evidence. Moreover, regulators in the same modules were frequently found to have related functions; for examples, Module M1 regulators were shown to have roles in vascular endothelial cell function, growth, and adhesion ${ }^{52-60}$. 
(1.18-fold higher, $\mathrm{P}=0.008)$, and 119 of its targets were adDEGs, of which $74 \%$ were AD-higher vs. and $A D$-association ${ }^{61,62}$, showed 1.37-fold AD higher ( $\left.P=8.8 \mathrm{e}-6\right), 53$ adDEG targets, with 100\% higher expression in AD; in Module 6, KLF4 previously linked to anti-inflammatory properties in endothelial cells ${ }^{64}$ showed 1.21-fold lower expression in AD ( $\left.P=8.2 e-4\right), 47$ (100\% AD-higher) adDEG targets, suggesting the activation of inflammatory response in AD. Not all regulators in each module were themselves DEGs, suggesting that some regulators may act through their collaboration with differentially-expressed regulators; for example, STAT3 and EP300 are significant adDEGs in module M1, but JUN, JUND, and GATA2 are not.

We used these regulator modules to partition their target adDEGs into sub-groups, mediated by distinct combinations of regulator modules (Methods). For cEndo AD-higher adDEGs, we found groups targeted by a single module (e.g. G4, G5, G6), and other groups targeted by multiple modules (e.g. G1, G2) (Fig. 3f), with distinct functional enrichments in common functional categories (Fig. 3g). For sistent with the known role of these regulators on chromatin structure maintenance ${ }^{65}$. Conversely, adDEG genes in Group1 were targeted by regulators from most modules (M1-M4, M6) and were significantly enriched in cytokine response, cell adhesion and transcription regulation. Genes in Group 5, targeted by Module 1 regulators, were enriched in lipid storage, interferon- $\beta$ response and blood-brain barrier maintenance. Genes in several groups (G3, G7, G8) were enriched in VEGFR signaling negative regulation, repression of endothelial cell proliferation, and apoptotic signaling pathways, suggesting endothelial injury response in $A D$, consistent with BBB breakdown.

We next sought to understand the mechanistic basis of $A D$-associated differences in vascular cell communication with glia and neurons in the neurovascular unit, that senses changing neuronal energy demands and adjusts local blood flow, and how this communication is altered in AD. We predicted bidirectional cell-cell communication between vascular and neuronal or glial cells using AD-associated covariation analysis of biologically-enriched gene modules across 409 individuals (Extended Data

Fig. 4a, Methods). We identified 301 higher interactions in AD, where one or both interacting modules showed higher expression in $A D$, and conversely 276 lower interactions in $A D$, where one or both interacting modules showed lower expression in $A D$, between vascular cell types (capillary and venule endothelial cells, pericytes, and fibroblast subtype1) and other brain cell types (inhibitory/excitatory neurons, microglia, oligodendrocytes, astrocytes and oligodendrocyte precursor cells) (Fig. 4a-b, Supplementary Table 9). We found that the communications from Per1 and cEndo to neurons, microglia and astrocytes dominate the higher cell-cell interactions in AD, while interactions from astrocytes and neurons to cEndo and Fib1 are mainly lower in AD. 
To characterize ligand-receptor signaling pathways potentially responsible for differential cell-cell communications in $A D$, we aggregated individual interactions mediated by the same ligand-receptor pair between vascular cell types and other main cell types, and classified the ligand-receptor pairs into 62 signaling pathways based on KEGG, ligand family, and receptor family annotations (Methods). We observed that the signaling pathways mediated by TGF- $\beta$, SPP1, BMP, ANGPTL and IL6 are significantly overrepresented for AD-higher interactions, while collagen and laminin, the major ECM proteins to form basement membrane of $\mathrm{BBB}^{66}$, are overrepresented for AD-lower interactions (Fig. 4c-d), suggesting the potential disruption of BBB structure in AD.

Moreover, we quantified AD-differential interactions in both "forward" and "reverse" communication directions for each pair of cell types and found that interactions between cEndo/Per1 and excitatory neurons/astrocytes show the most interactions (Extended Data Fig. 4b), suggesting the important roles of the neurovascular unit in AD pathology. We then built ligand-receptor networks for top three cell-cell pairs (cEndo-Ex, cEndo-Astro, and Per1-Ex) to highlight specific signaling pathways and ligand-receptor pairs (Fig. 4e-g). For example, EFNA1, a member of the ephrin family which inhibits axonal growth via EPH signaling ${ }^{67}$, increasingly expressed by cEndo in AD interacts with multiple receptors expressed in excitatory neurons (Fig. 4e), suggesting that endothelial cells may mediate axonal growth disruption in AD. TGFB1, which has been shown to exacerbate BBB permeability and regulate pericyte inflammatory response ${ }^{68,69}$, is highly expressed in capillary cell types in AD (cEndo and Per1), and mediates communications with excitatory neurons (Fig. 4e,g), suggesting the important roles of TGF- $\beta$ in reactive oxygen species generation, amyloid- $\beta$ accumulation and neuronal dysfunction during $A D$ pathogenesis ${ }^{70}$. BMP6 mediates the AD-higher interactions between pericyte1 and excitatory neurons, suggesting its function in pericytes governing impaired neurogenesis in $A D^{71}$. The $A D-l o w e r$ interactions between astrocyte and capillary endothelial cells mediated by EGF signaling pathways suggests inhibition of capillary endothelial proliferation in $A D^{72}$ (Fig. 4f).

\section{AD GWAS loci linked to brain vascular adDEGs}

We next sought to gain insight into how $A D$ risk loci may lead to vasculature breakdown at the molecular level, by integration of our adDEGs with AD-associated loci from genome-wide association studies (GWAS) $)^{73-75}$ (Fig. 5a), to predict their candidate target genes and directionality of effect (higher or lower expression in AD) (Fig. 5b), their cell types of action (Fig. 5c,d), and the direct (Fig. 5a-g) or indirect (Fig. $\mathbf{5 h - j}$ ) mechanisms through which they can lead to vascular gene expression changes. AD-associated variants in 113 loci $^{76,77}\left(\mathrm{P}<10^{-5}\right)$ (Fig. 5a) proximal to 125 vascular adDEGs (Fig. 5b, 
capture (Hi-C) looping ${ }^{78-81}$, correlation-based enhancer-gene links ${ }^{82}$; brain/heart/muscle-specific eQTLs at tissue-level resolution ${ }^{83}$; and vasculature cell-type specific single-cell eQTLs (sc-eQTLs) as we reported for cell-type-specific adDEG enrichments, including cholesterol transport, sterol homeostasis, regulation of endothelial cell and vascular associated smooth muscle cell migration, regulation of amyloid precursor protein catabolic process and IL6 mediated signaling (Fig. 5g, Supplementary Table 10), and we highlight some examples next.

Twenty-one GWAS loci were associated with lipid and cholesterol metabolism adDEG genes, consistent with broad dysregulation of brain cholesterol homeostasis in $A D^{84}$, including several notable examples. First, RORA (Extended Data Fig. 5b), a lipid-sensing nuclear receptor, was broadly lowlyexpressed (in cEndo, vEndo, pericytes, fibroblasts), was linked (by endothelial Hi-C chromatin loops, muscle eQTLs, SMC sc-eQTLs, pericyte sc-eQTLs) to its own AD-associated intronic variant (rs3784609), and was previously-shown ${ }^{85}$ to regulate pathological retinal angiogenesis by repressing inflammation repressor SOCS3, which is indeed also highly expressed in capillary and venule endothelial cells in AD in our data. Second, ABCA1 (Extended Data Fig. 5c), a cholesterol transporter, was linked (by correlation-based enhancer-gene links, muscle and fibroblast eQTLs, sc-eQTLs in endothelial, fibroblast, SMCs) to four AD-associated intronic variants, and was highly expressed in pericytes, which increases pericyte cholesterol efflux to $\mathrm{ApoE}^{86}$, and lowly expressed in fibroblasts, which reduces amyloid- $\beta$ deposition and clearance through $A p o E^{87,88}$. Third, SCARB1 (Extended Data Fig. 5d), a cholesterol exchange regulator, was linked (by endothelial Hi-C, endothelial sc-eQTLs, muscle eQTLs) to its own AD-associated intronic variant (rs78194510), was highly expressed in capillary endothelial cells and pericytes in $A D$, and was shown to mediate HDL signaling in endothelial cells and regulate astrocyte-A $\beta$ and SMC-A $\beta$ interactions ${ }^{89,90}$.

Nine AD GWAS loci were associated with immune response, insulin secretion and neurodegenerative pathogenesis, including several notable examples. First, IL6 and its receptor IL6R were highly expressed in capillary endothelial cells of $A D$ individuals, suggesting immune response of endothelial cells may be a prominent feature of AD blood vessels ${ }^{91,92}$. Second, MYRIP, an insulin secretion regulator, was associated with $100 \mathrm{~kb}$ upstream variant rs9832461 through $\mathrm{Hi}-\mathrm{C}$ loop in endothelial cells and enhancer-gene correlation in endothelial cells and brain, and was lowly expressed in capillary endothelial cells of $A D$ (Extended Data Fig. 5f), consistent with the expression change in $A D^{93,94}$, and suggesting potential dysregulation of insulin signaling ${ }^{95}$. Third, PFDN1, encoding one subunit of prefoldin complex associated with $A D$ pathogenesis ${ }^{96,97}$ and showing lower expression in venule SMCs of $A D$ patients, was linked to AD variant rs11168036 through enhancer-gene correlation in brain, heart, and muscle, Hi-C loop in endothelial cells and eQTLs in muscle and endothelial cells (Extended Data Fig. $\mathbf{5 g}$ ), suggesting the dysregulation of protein folding machinery in cerebrovascular cells in AD. 
Second, we searched for indirect genetic evidence for adDEGs whose upstream regulators were directly linked to $A D$-associated variants (Fig. 5h). Five of our previously-predicted (Fig. 3a-b) upstream transcription factors (YAP1, TCF7L2, NFIC, ETS1, DACH1) were directly linked to AD-associated variants, a 2.9-fold enrichment, given only 33 TFs lie in AD-associated loci (Fisher's exact test, pvalue=0.04). The first four TFs were also predicted to be upstream regulators of cell-type-specific marker genes in our earlier analysis (Fig. 1e). These were linked to AD-associated SNPs through diverse lines of evidence: YAP1 through sc-eQTLs in endothelial cells and pericytes; ETS1 through HiC loop in endothelial cells; TCF7L2 through EpiMap promoter-enhancer correlation in endothelial cells and heart, HiC loop in endothelial cells, and eQTL from GTEx in heart; and NFIC and DACH1 through $\mathrm{HiC}$ loop in endothelial cells. These AD-associated adDEG regulators targeted 559 vascular adDEGs across five cell types (Fig. 5h, Supplementary Table 11), which showed biologically-meaningful enrichments, including: for YAP1, cell migration regulation, angiogenesis and extracellular matrix organization across multiple cell types; for ETS1 cytokine and growth factor stimulus response in capillary endothelial cells, consistent with prior work ${ }^{17,19-21}$; for TCF7L2, a known master regulator in vascularization, SMCs plasticity, glucose homeostasis and insulin production and processing ${ }^{98-100}$ in cEndo, pericytes and fibroblasts.

Third, we searched for indirect genetic evidence for vascular adDEGs downstream of ligand-receptor pairs, whose ligands are directly linked to $A D$-associated variants, and differentially expressed in $A D$ (adDEGs) in nonvascular cells (neurons, astrocytes, oligos, OPCs, microglia), thus potentially leading to vascular cell dysregulation through ligand-receptor signaling pathways. We used our previously-annotated correlated module pairs (Fig. 4, Extended Data Fig. 4) that showed coordinated expression differences in AD between vascular and neuronal or glial cell types, and searched for AD-linked adDEG ligands indicative of potential genetic effects. Among our 577 previously-defined module pairs, we found 54 pairs (24 AD-higher and 30 AD-lower) with ligands proximal to AD-associated genetic loci (Fig. 5i), implicating 611 vascular adDEGs, which are linked to $12 \mathrm{AD}$-associated ligands and 13 receptors (in 18 different ligand-receptor pairs). The 12 AD-associated ligands represent a significant enrichment over expectation (odds ratio $=2.52$, p-value $=0.0016$, Fisher's exact test). Of these 12 ligands with proximally linked AD-associated variants, 7 showed sc-eQTL linking evidence, 3 showed tissuelevel brain-eQTLs evidence from GTEx, 7 showed Hi-C loop linking evidence, including 6 with multiple lines of evidence (Fig. 5i, col. 2). Their downstream genes were enriched in at least 17 different biological functions (Fig. 5j, Supplementary Table 12). For example, AD-associated rs442495 (Pvalue $=3 e-11$ ) in Chr15p13 was linked (through HiC loop and tissue-eQTLs) to ADAM10, a key modulator of dendritic spine formation and AD pathology ${ }^{101}$, an adDEG differentially expressed in multiple cell types (oligodendrocyte, astrocyte, excitatory and inhibitory neurons, and OPCs), that binds three adDEG receptors (IL6R ${ }^{102}, \mathrm{NOTCH} 1{ }^{103}, \mathrm{TSPAN}^{14}{ }^{104}$ ), all differentially expressed in capillary endothe- 
downstream of ADAM10-IL6R signaling activates high expressed endothelial genes enriched in lipid storage and response, immune response, and cell proliferation. Interacting with NOTCH1 induces genes involved in cell migration and proliferation. We also observed APOE, the strongest AD genetic associated gene and showing higher expression in microglia and lower expression in astrocyte, interacts with LRP6 ${ }^{105}$ to regulate the expression of genes in pericytes significantly enriched for cell junction and cell migration, which is lower in $A D$.

Taken together, we found 1,010 of 2,676 vascular adDEGs in AD can be associated with AD genetics using cis, trans-, or signaling regulatory mechanisms (Extended Data Fig. 5a, h, Supplementary Table 13). The expression and transcriptional differences in vascular cell types in the context of AD point out that the effects of genetic risk factors on cerebrovasculature may also contribute to the pathogenesis of AD through the intracellular dysfunction and underlying intercellular communications with neural,

\section{APOE4-associated transcriptional differences and cognitive decline}

The apolipoproteinE (APOE) genetic locus is the largest genetic risk factor of late-onset $A D$ with an increased risk for APOE $\varepsilon 4$ allele carrier (E4) relative to the common $\varepsilon 3$ allele ${ }^{106}$ (E3), capturing more $A D$ heritability than all other known markers combined ${ }^{107}$. APOE $\varepsilon 4$ has been previously reported to exacerbate $\mathrm{BBB}$ breakdown and pericyte dysregulation ${ }^{108}$, which are thought to contribute to cognitive impairment ${ }^{109-111}$. To elucidate the molecular mechanisms and vascular cell types potentially mediating the effects of APOE $\varepsilon 4$ on BBB dysfunction and cognitive decline, we searched for APOE-genotypeassociated differentially expressed genes (apoeDEGs) between cells of APOE $\varepsilon 3 \mid \varepsilon 3$ homozygous individuals $(E 3, N=251)$ vs. carriers of one or two APOE $\varepsilon 4$ alleles $(E 4, N=101$ heterozygous $\varepsilon 3 \mid \varepsilon 4$ and $\mathrm{N}=7$ homozygous $\varepsilon 4 \mid \varepsilon 4$ ), controlling for other pathological and demographic variables, including $A D$, age, sex, PMI, Parkinson's, Lewy Body, VCID, and cognitive decline (see Methods). As the vast majority of $\varepsilon 4$ carriers are heterozygous in the population (and in our cohort), we do not focus on homozygous carriers here.

401

We found 2,482 apoeDEGs (Fig. 6a, Supplementary Table 14, Methods), which were mostly evenly distributed across cell types (120 APOE4-higher and 120 APOE4-lower on average) and similar in count to the number of adDEGs. While only a median of $4 \%$ of APOE-differential genes were shared with $A D$-differential genes, reflecting the additional contributors to AD beyond the APOE genotype, the overlap between apoeDEGs and adDEGs was highly significant for a subset of cell types. For capillary endothelial cells (cEndo), 36\% of APOE4-higher apoeDEGs were also adDEGs (12-fold enrichment, $\mathrm{P}=10^{-32}$, Fisher's exact test) and $21 \%$ of APOE4-lower apoeDEGs were also adDEGs (12-fold, $\mathrm{P}=$ 10 $^{-}$ 
411 To gain more insights on the specific biological functions affected by the APOE $\varepsilon 4$ genotype, and thus 412 potential therapeutic hypotheses against AD-associated BBB breakdown, we searched for enriched 413 biological pathways in E4-higher and E4-lower genes in each of these cell types (Fig. 6b, Supple414 mentary Table 15). In capillary endothelial cells, E4-lower genes were significantly enriched in 415 transport across blood-brain barrier (e.g. ABCB1, ABCG2, SLCO2B1, SLC48A5), cell junction organi416 zation (APBB2, CAPZA1, SDK1, PPFIBP1) and regulation of sprouting angiogenesis (JMJD8, KLF4, $417 \mathrm{KLF} 2$ ). and in pericytes E4-lower genes were enriched in cell migration regulation, transport across 418 BBB (ATP1A2, SLC6A13, SLC19A1, SLC6A1), and cell-cell junction maintenance (CSF1R, TJP1) 419 (Fig. 6b), in agreement with reports of ApoE $\varepsilon 4$ individuals showing reduced cerebral blood flow ${ }^{112}$, 420 and cerebrovascular abnormalities ${ }^{113}$. APOE4-higher genes were significantly enriched for cytokine 421 response in both capillary endothelial cells and pericytes, negative regulation of cell migration in capil422 lary endothelial cells, and apoptotic process and positive regulation of endocytosis in pericytes (Fig. 423 6b), consistent with increased risk for neurodegeneration and pathology in APOE $\varepsilon 4$ individuals.

We next evaluated the correlation of all apoeDEGs with cognitive decline in all cell types, and found that higher expression of E4-higher genes was primarily associated with cognitive decline across all cell types, while conversely higher expression of E4-lower genes was primarily associated with cognitive resilience (Fig. 6c, Extended Data Fig. 6a). This effect was strongest for cEndo $\left(p<10^{-22}\right)$, vEndo $\left(p<10^{-11}\right)$, Fib1 $\left(p<10^{-11}\right)$, and Per1 $\left(p<10^{-8}\right)$, with substantial and highly-significant differences in cognitive loss correlation between E4-higher vs. E4-lower genes. Our results are consistent with previous findings suggesting capillary pericytes might mediate the effect of APOE4 on cognitive decline ${ }^{111}$, and indicates that capillary endothelial cells and fibroblasts subtype 1 might play equally or even more important roles, based on both the overlap of apoeDEGs and adDEGs, and the highly significant correlation with cognitive decline in both cell types.

We further identified cognitive-decline-correlated differentially-expressed genes (cogDEGs) for each vascular cell type, for both APOE3 and APOE4 individuals (Fig. 6d, Supplementary Table 16, Methods), distinguishing "decline-lower" cogDEGs that are negatively-correlated with cognitive decline vs. "decline-higher" genes that are positively-correlated with cognitive decline. APOE4 individuals showed more cogDEGs than APOE3 individuals (>1.5-fold enrichment) for cEndo, Per1, Fib1, and vEndo (Fig. 6d), especially for decline-higher genes (2.1-fold), suggesting specific cerebrovascular cell types that might mediate the contribution of the APOE $\varepsilon 4$ allele on cognitive decline. Comparing APOE3-specific cogDEGs vs. APOE4-specific cogDEGs, we found that the two sets were largely distinct, with only $\sim 5 \%$ of cogDEGs in common between APOE4 and APOE3 (Fig. 6e), suggesting distinct transcriptional changes and potential specific mechanisms of cognitive decline between $\varepsilon 3$-only vs. $\varepsilon 4$ carriers; however this small number of shared cogDEGs was significant in Fib1, Per1, and cEndo. 
To further investigate the functions of APOE3 vs. APOE4 cogDEGs, we performed Gene Ontology enrichment analysis for E3-specific, E4-specific, and E3-E4-shared cogDEGs, both decline-higher and decline-lower, in both capillary cell types (cEndo and Per1) (Fig. 6f, Supplementary Table 17). For cEndo decline-higher cogDEGs, APOE4-specific enrichments included lipid and cytokine response, apoptotic process and negative regulation of growth, and APOE3 enrichments included vascular transport, negative regulation of cell migration, differentiation and cell matrix adhesion. For cEndo decline-lower cogDEGs, APOE4-specific enrichments included blood vessel development, positive regulation of BMP signaling and neurotransmitter transport regulation, and APOE3 enrichments included positive regulation of endothelial cell migration and extracellular structure organization. For Per1 decline-higher cogDEGs, APOE4-specific enrichments included cytokine response, DNA damage response and apoptosis, and APOE3 enrichments included negative regulation of SMC migration and Notch signaling, and autophagy. For Per1 decline-lower cogDEGs, APOE3 enrichments included lipid and chemical homeostasis and cell junction assembly, whereas APOE4-specific decline-lower cogDEGs were enriched for BBB-related functions (BBB maintenance, Notch signaling, contraction regulation), suggesting that APOE $\varepsilon 4-d e p e n d e n t$ cognitive decline may be primarily mediated by Per1 pericytes (Fig. 6f).

\section{Discussion}

In this study, we profiled and analyzed the transcriptome of 22,514 single nuclei, identified the molecular signatures and upstream regulators of eleven brain vascular cell types, and characterized regionspecific expressed genes and pathways in 428 AD and control individuals. We identified 2,676 AD-associated differentially expressed genes (adDEGs) with strong cell-type specificity, including low expression of some canonical cell-type markers and key genes for BBB integrity in AD (e.g. pericyte marker PDGFRB ${ }^{4,29}$ ), highlighting the specialized functions of vascular cell types in the maintenance of the brain-blood barrier and its dysregulation in disease. These adDEGs were enriched in multiple biological pathways broadly across cell types, including immune response, insulin response, and vasculogenesis, specifically in single cell types, including synaptic transmission in pericytes and Notch signaling in endothelial cells. These results suggest dysregulation of both common functions and cell typespecific pathways, providing potential clues for both global and targeted therapeutic directions aiming at the AD cerebrovasculature.

We also predicted upstream regulators of adDEGs, and grouped them into collaborative regulator modules, potentially driven by primary regulators. Using these modules, we clustered targeted adDEGs into groups specified by higher-order combinations of these modules, each of which was associated with distinct biological functions. Our analysis provides a general framework for understanding how regulators collaboratively control dysfunctional gene programs in disease and may aid in prioritizing for therapeutic targets to restore the function of AD-differential genes, developing iPSC-derived vasculature, and planning perturbation experiments. 
481

482

483

484

485

486

487

488

489

490

491

492

493

494

495

496

497

498

499

500

501

502

503

504

505

506

507

508

509

510

511

512

513

514

We next investigated differential cell-cell communications of neurovascular units in AD. Methodologically, we introduced a new computational framework by combining covariation analysis of gene co-expression modules between cell types across 428 individuals with ligand-receptor pairs and their corresponding signaling pathways. We identified 577 AD-differential cell-cell communications (301 ADhigher and 276 AD-lower) and investigated the responsible signaling pathways. Notably, we found that collagen and laminin, the basic components of the basement membrane of BBB structure, were significantly mediating AD-lower interactions, suggesting the BBB breakdown in AD. Signaling pathways including TGF- $\beta$, SPP1, BMP and IL6 were overrepresented in AD-higher interactions, suggesting the potential neuronal dysfunction, $A \beta$ accumulation and immune response of NVU in $A D$. The dynamics of multi-cellular interactions in AD calls attention to the development of multicellular in vitro systems and provides a specific point of view to therapy in the future.

Moreover, our study yielded insights on interpreting AD genetic variants from genome-wide human genetics studies in cerebrovascular cell types. We innovatively proposed and studied three types of mechanisms to understand how $A D$ variants are associated with vascular differential genes in $A D$ : (1) direct regulation in a "cis" way (125 adDEGs), (2) indirect regulation in a "trans" way (559 adDEGs through five AD-GWAS-proximal regulators) and (3) indirect regulation through intercellular signaling pathways (611 adDEGs through 12 differentially expressed AD-GWAS-proximal ligands). Altogether, we observed that 1,010 of $2,676(37.7 \%)$ adDEGs could be associated with AD genetics, suggesting the importance of understanding the BBB dysregulation in AD from the genetic perspective and provides a paradigm to be widely applied in multiple scenarios including other cell types in AD and other diseases.

Finally, our study shed light on the molecular and cellular basis to understand the association of cerebrovasculature with APOE4-associated cognitive decline. We found APOE4 highly expressed genes in cEndo, vEndo, Per1 and Fib1 were significantly and positively correlated with cognitive decline, suggesting that these could be the major cell types associated with APOE4-dependent cognitive impairment. We further observed that cognitive-decline-associated transcriptional differences were APOE genotype specific. For example, the significant enrichment of BBB functions in cognitive-decline-lower genes was specific to APOE4 pericytes, demonstrating that APOE $\varepsilon 4$ allele leads to cognitive decline through BBB dysfunction at both cellular and molecular levels. This offers the potential therapeutic targets regarding BBB functions in specific APOE genotypes on cognition impairment. Given the limited number of vascular cells, especially for rare cell types in APOE2 and APOE4, further targeted studies are needed to comprehensively understand the association and causality among APOE genotype, BBB function, cognitive decline and AD.

Overall, our multi-region molecular atlas of differential human cerebrovasculature genes and pathways 
bioRxiv preprint doi: https://doi.org/10.1101/2022.02.09.479797; this version posted February 10, 2022. The copyright holder for this preprint (which was not certified by peer review) is the author/funder. All rights reserved. No reuse allowed without permission.

515 in $A D$ provides an important foundation for guiding $A D$ therapeutics, especially for early-stage inter516 ventions where the BBB is increasingly recognized to play a central role. 


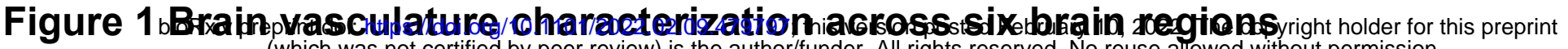
a
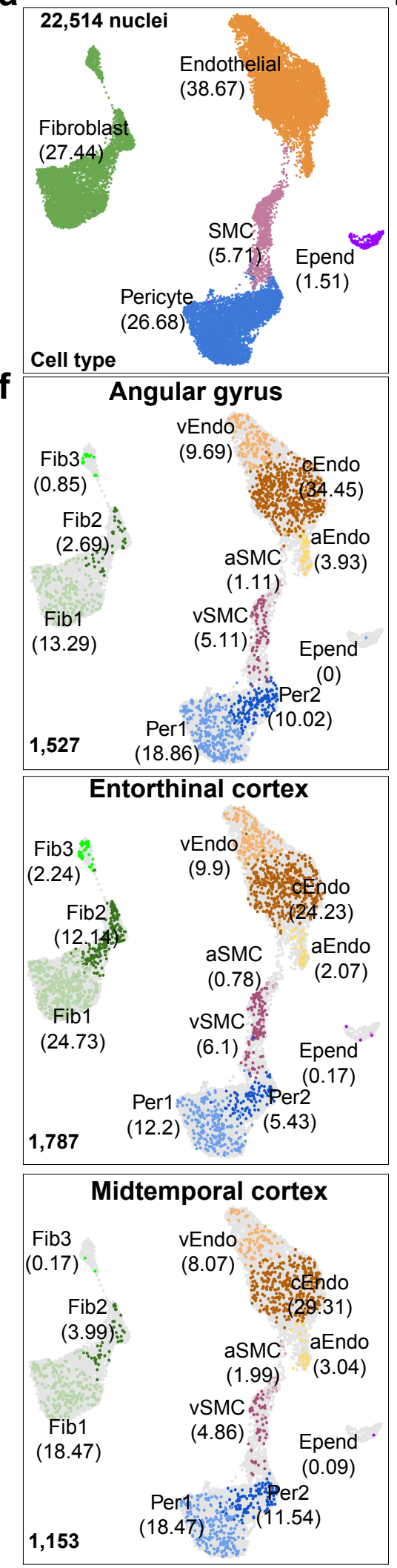

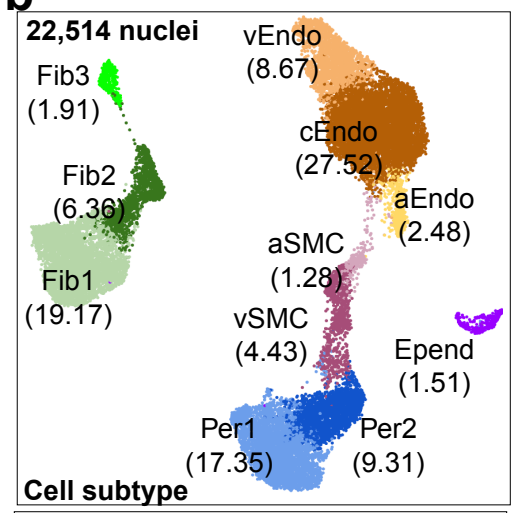

Cell subtype
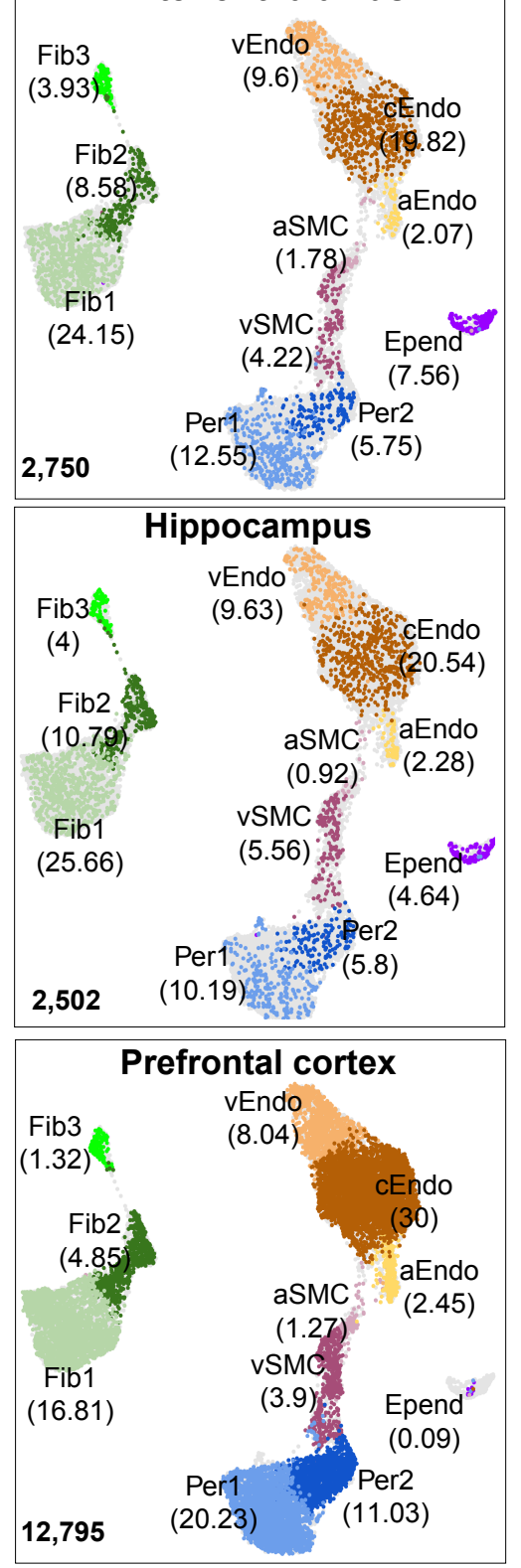

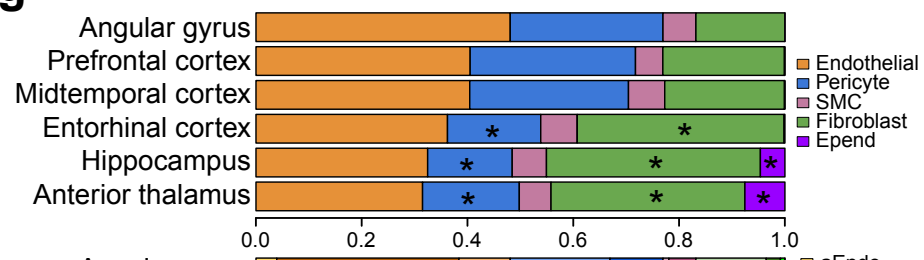

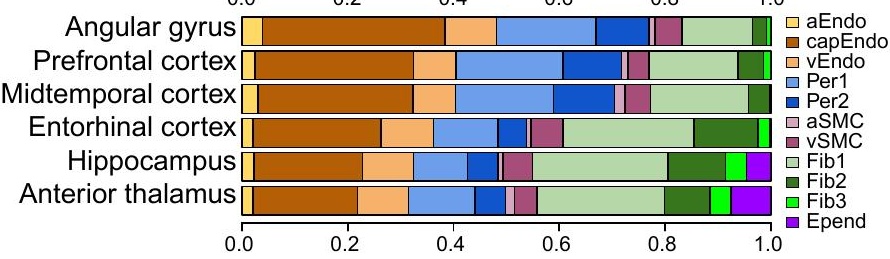

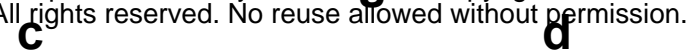
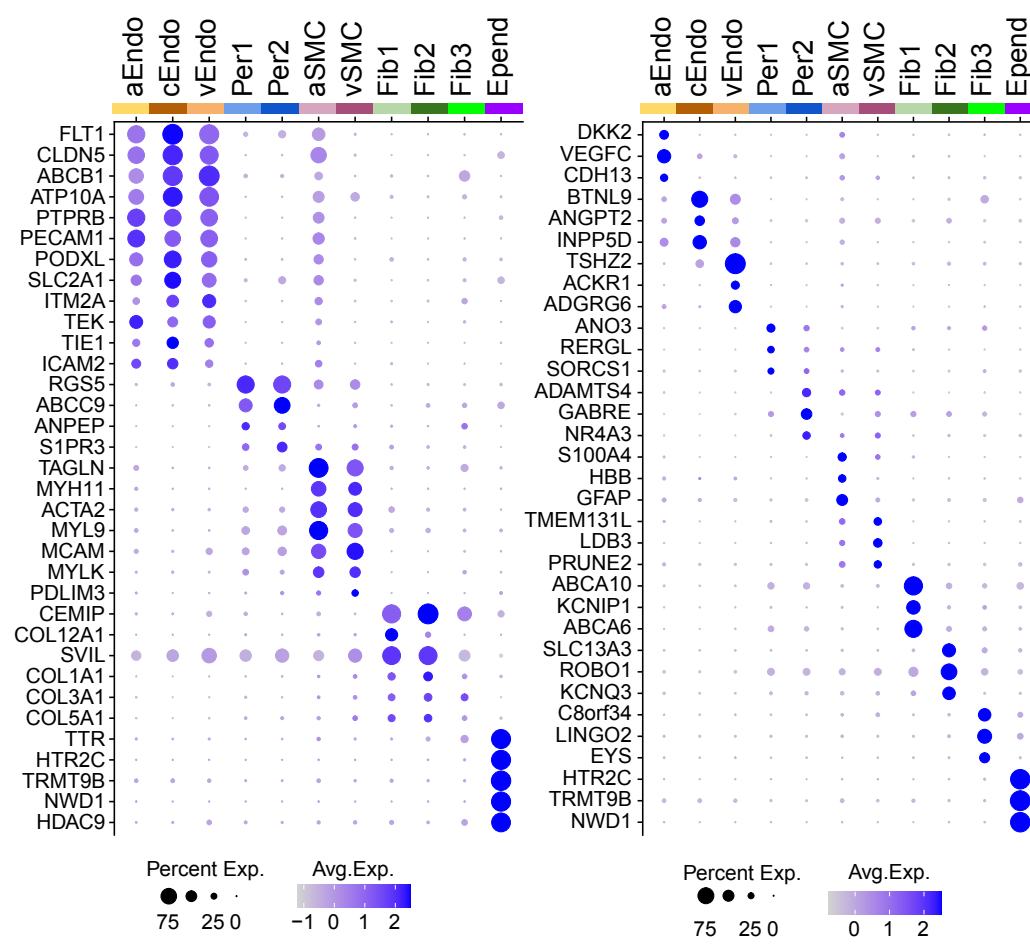

e

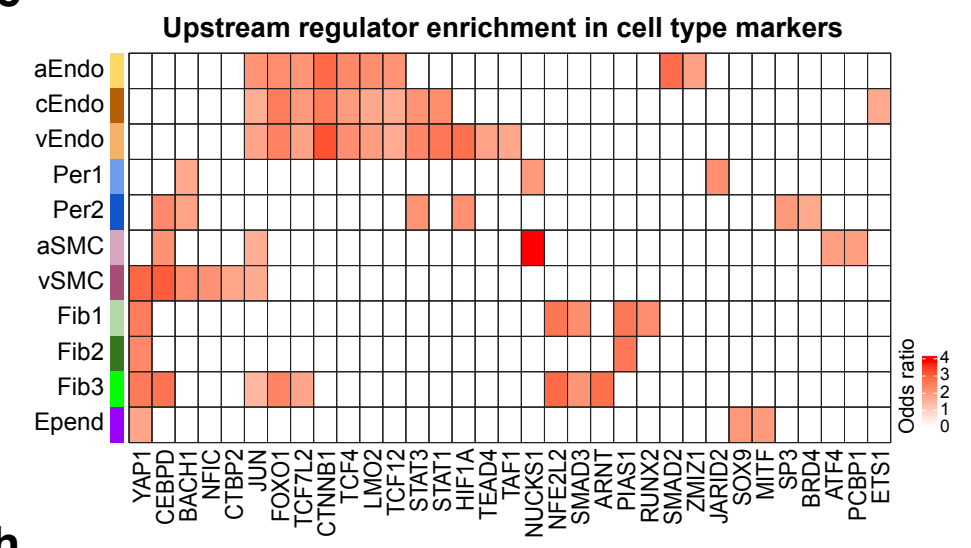

h
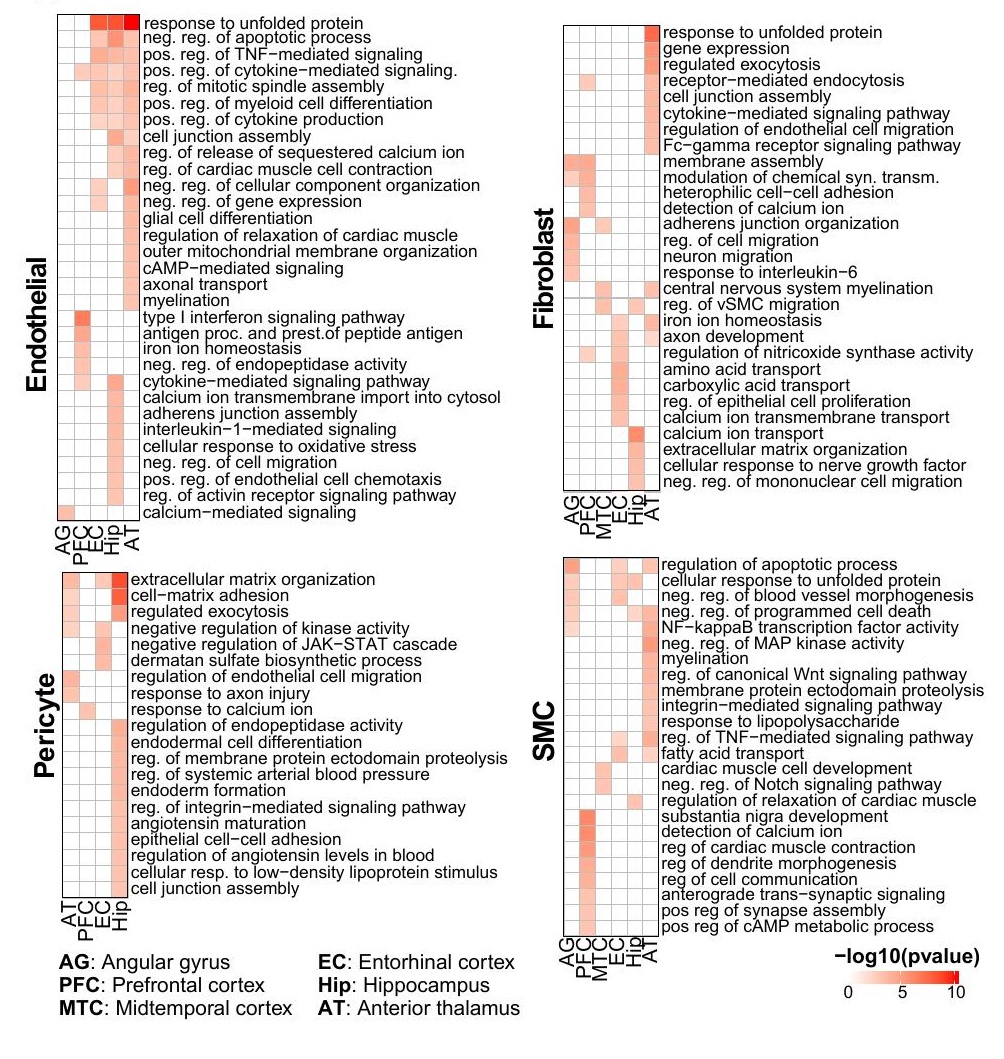
517 Figure 1. Brain vasculature characterization across six brain regions. a-b. UMAP of 22,514 in sil-

518 ico sorted brain vascular nuclei from postmortem tissues labeled by cell type (a) and cell subtype (b),

519 the percentage of cells in each cell is shown. c-d. Top markers for vascular cell types (c) and cell sub520 types (d). e. Heatmap to show the enrichment of upstream regulators for cell subtype markers. $\mathbf{f}$.

521 UMAP of vascular nuclei for each brain region. g. Distribution of cell fraction across six brain regions. * 522 represents the significant enriched cell types in specific regions by the Wilcoxon rank test $p$-value $523<0.01$. h. Representative enriched Gene Ontology biological processes of brDEGs for each cell type. 
524 Figure 2. Cell-type-specific brain vasculature differences in AD. a. Overview of adDEGs in each

525 cell type. From left to right panels, it shows the numbers of cells and expressed genes, the number of 526 lower/higher adDEGs in AD, and the heatmap with each gene in column and each cell type in row. $\mathbf{b}$.

527 The number and significance of adDEGs overlap between cell types in both directions (upper triangle: 528 AD-higher adDEGs; lower triangle: AD-lower adDEGs). c. Top5 highly/lowly expressed DEGs in AD in 529 each cell type. The highest effect size for each gene is colored by the cell type. $\mathbf{d}-\mathbf{g}$. Enriched Gene 530 Ontology biological processes in AD-higher (d) and AD-lower (e) adDEGs in capillary endothelial cells, 531 AD-higher (f) and AD-lower (g) adDEGs in Per1. h-i. Representative images (h) and quantification (i) 532 of INSR gene expression in $\mathrm{CD} 31^{+}$endothelial cells from control and AD prefrontal cortex tissue. j-k. 533 Representative images (j) and quantification (k) of APOD gene expression in $\mathrm{GRM}^{+}$pericytes from 534 control and AD prefrontal cortex tissue. 

118 regulators upstream of $A D$ Dhigh DEGs

SETDB1 Epend

JARID2 PAS1 1 TOP2B MECOM CEPPE USMC ZEEB

CRTC3 Fib1 TOP2B MECOM CBPPS VSMC ZEBB1

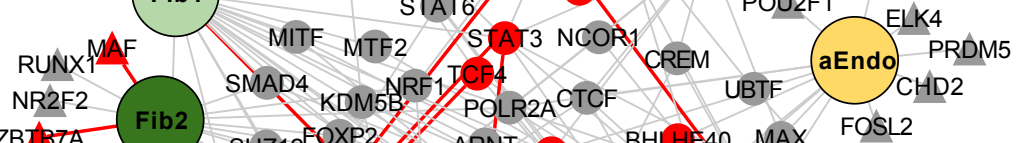

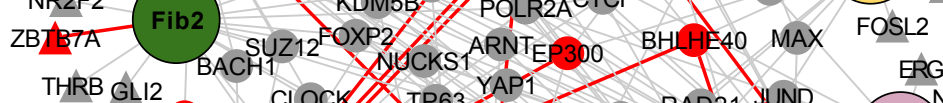

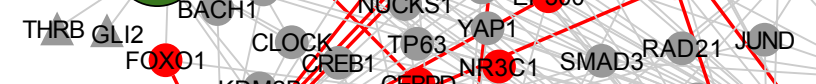

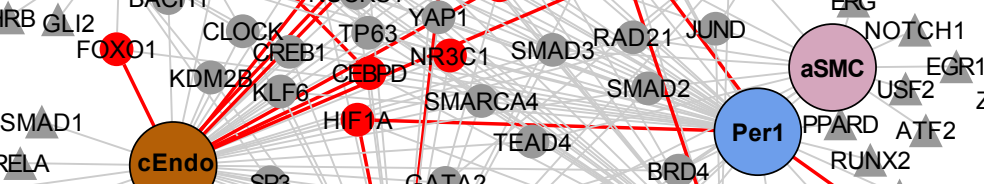
ETS1 RELA CENDO SP3 STAT1 GATA2

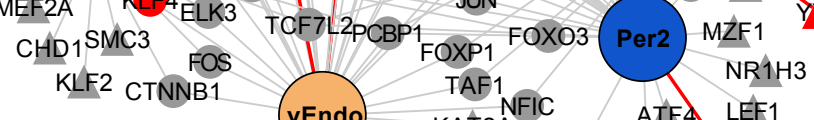
LMO2 VEndo KAT2A NEIC ACOR3 ATFA LEF1 HSFF DOP 1A ZKSSCAN1 KAT2B ZNF148 $\mathrm{ESR}_{\mathrm{PBX} \times 3}$
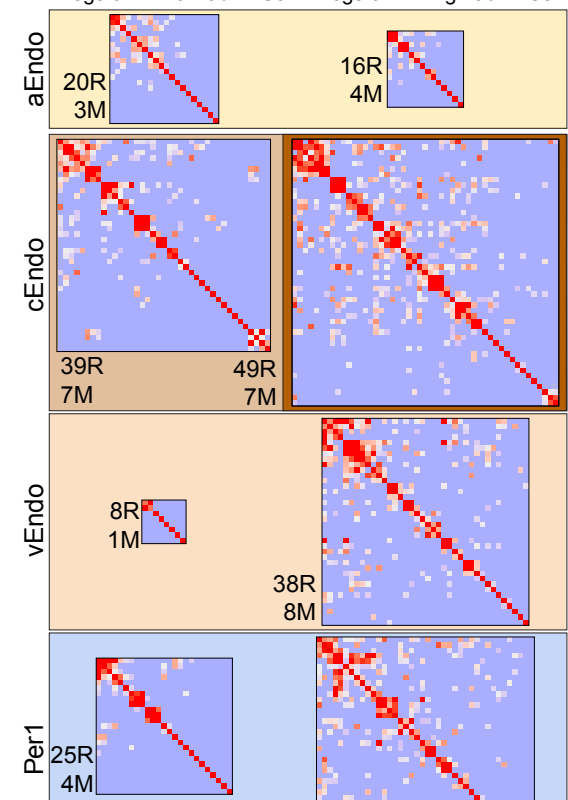

$38 \mathrm{R}$
$8 \mathrm{M}$

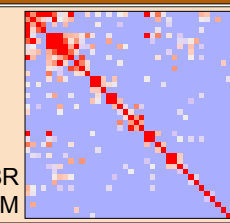

OR
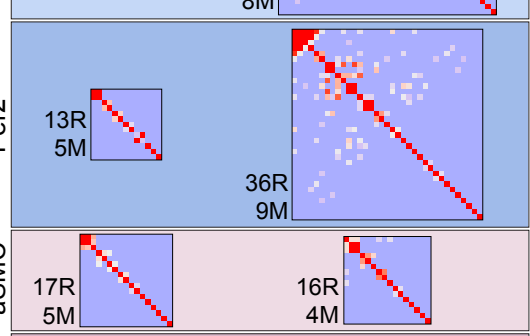

$9 \mathrm{M}$

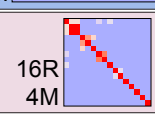

$5 \mathrm{R}$
$1 \mathrm{M}$

$\mathrm{M}$

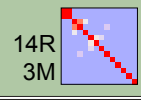

욘 17

$3 \mathrm{M}$

OM

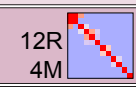

'4R'=4 Regulators
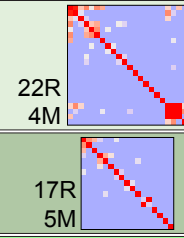

$10 \mathrm{R}$
$1 \mathrm{M}$

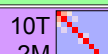

$2 \mathrm{M}$

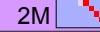

'M'=2 Modules with >1 Reg.
C Regs of AD-low adDEGs Regs of AD-high adDEGs d

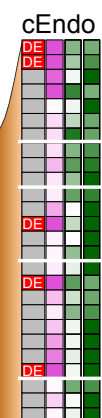

Regulators of cEndo AD-high adDEGs

$f$

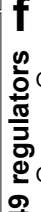

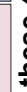

要

ס

\$ٕ

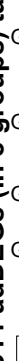

BP2 KDM2B

RELA PRDM1

STAT2 JUN

$\mathrm{LMO} 2 \mathrm{FOXO}_{3}$

SMAD2 GATA2

TCF12

CTNNB1
1
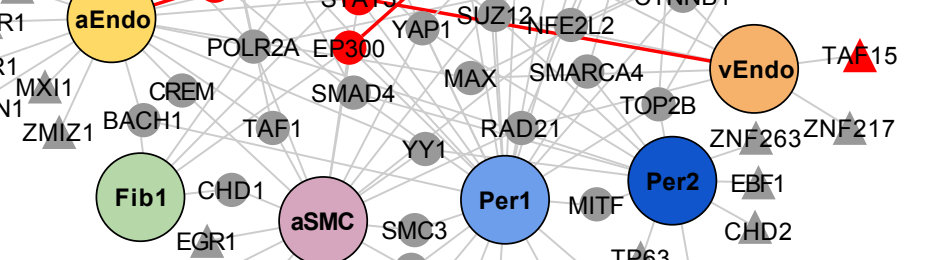

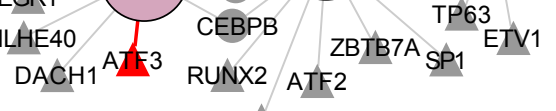

PCBP1

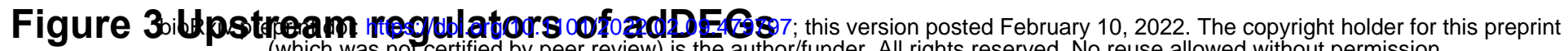

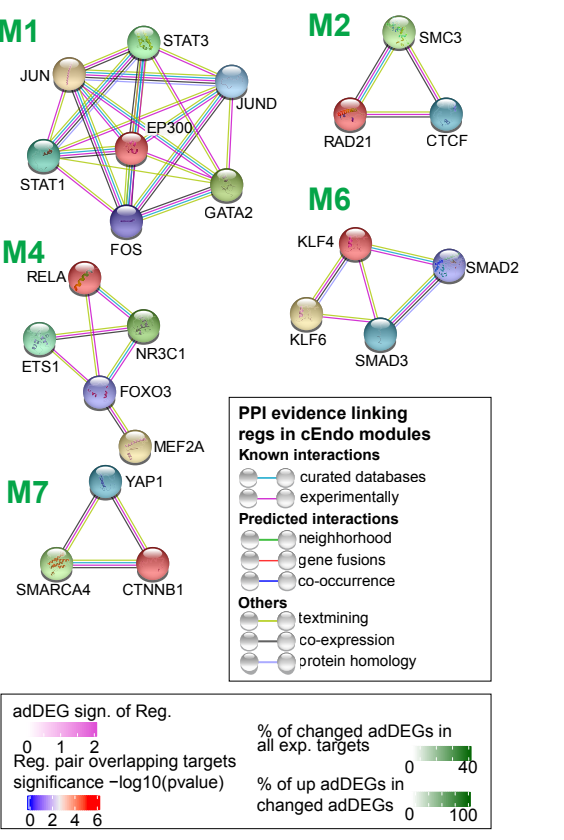

M6

PI evidence linking

experimentally

of changed adDEGs in

$\%$ of up adDEGs in

changed adDEGs $0 \quad 100$

cytokine stimulus M4 regulation of cell adhesion

growth hormone receptor signaling
positive regulation of phagocytosis

posive mula phagocy

negative regulation of intrinsic apoptotic signaling pathway

$\begin{array}{ll}\text { M2 } 2 & \text { neg. reg. of VEGR signaling; extrinsic apoptotic signaling } \\ \text { Minositol lipid-mediated signaling; cholesterol transport }\end{array}$

cellular response to cytokine stimulus

celluar matrix assembly

negative regulation of extracellular matrix organization

regulation of lipid storage
activation of GTPase activity

M1 activation of interferon-beta

maintenance of blood-brain barrier

negative regulation of response to cytokine stimulus

3 regulation of gene silencing by miRNA

regulation of cell division

3 negative regulation of endothelial cell proliferation

cellular response to metal

negative regulation of growth
not

Gene Ontology Biological Processes

enriched in each group of cEndo adDEGs

co-targeted by common TF modules

- Regulator modules showing abundant targeting in group 
535 Figure 3. Upstream regulators of adDEGs. a-b. Regulator-cell type networks in AD-higher (a) and

536 AD-lower (b) adDEGs. The large nodes represent cell types. Triangle nodes represent cell type spe-

537 cific regulators. Grey nodes represent regulators with expression. Red nodes represent differentially

538 expressed regulators in at least one relevant cell type. Red edges represent the corresponding differ-

539 entially expressed regulators in relevant cell types. c. Regulator modules of all adDEGs sets. The size

540 of heatmaps reflects the number of regulators. d. Regulator modules of AD-higher adDEGs in capillary

541 endothelial cells. Seven modules were labeled by a green rectangle. The first column on the left shows

542 if the regulator is significantly differentially expressed. The second column shows the level of differen-

543 tial significance represented by $-\log 10(p$-value). The green columns show the percentage of changed

544 targets for each regulator and the percentage of AD-higher adDGEs in changed targets. e. Protein-

545 protein interaction networks from STRING for each regulator module. f. Eight groups of adDEGs that

546 are targeted by regulators shown in (d). The gray shaded block highlights the regulation of specific

547 regulator modules on the gene group, which is also shown on the left column of $(\mathbf{g})$. The first column

548 on the left shows the effect size of adDEGs. The second column shows the level of differential signifi-

549 cance represented by -log10(p-value). The third column shows the number of regulators for each tar-

550 get. g. Enriched Gene Ontology biological processes of each group of genes in (f). 


\section{Figure 4. Dynamics of cell-cell communications between vascular cell types and neuron/glial}

552 cells in AD. a-b. Higher (a) and lower (b) interactions mediated by ligand-receptor signaling between 553 vascular cell types and neuron/glial/microglial cell types in AD. c-d. Ligand-receptor pairs (row) in each 554 pair of cell types (column) for higher (c) and lower (d) interactions in AD. The signaling pathway is also 555 shown on the right. The star in the signaling pathway column indicates that the signaling pathway is 556 significantly enriched. e-g. Top 3 pairs of interacting cell types: cEndo-Ex, cEndo-Astro, and Per1-Ex. 557 Ligand-receptor interactions with direction are shown in the network. The color of nodes represents 558 the cell type. Red edges represent AD-higher interactions, while blue edges are for AD-lower interac559 tions. 
560 Figure 5. AD GWAS loci linked to brain vascular adDEG. a-g. Direct (cis) regulated adDEGs by

561 AD-associated variants. Shown are the AD GWAS loci with subthreshold of -log10(p-value) as 5 asso562 ciated with significant adDEGs (a-b), along with the cell-type in which the largest differential expres563 sion occurs (c), a heatmap to present all differential expression across all cell types (d), the genomic 564 annotation for SNPs (e left), the distance between variant and adDEG transcription starting site (TSS) 565 (e middle), the rank of adDEG among all associated genes of the specific variant (e right), a heatmap 566 with four pieces of linking evidence (gene-enhancer correlation from EpiMap, tissue eQTL from GTEx, 567 sc-eQTL, and Hi-C data) (f), and a heatmap to highlight the enriched functions of adDEGs (g). h. Sum568 mary of $A D$-associated transcription factors: AD-variants, linking evidence, the number of targets in 569 adDEGs (up: top; down: bottom) and representative functions of these targets. i. Summary of AD-as570 sociated ligands: AD-variants, linking evidence, ligand, receptor, signaling pathway, sender cell type, 571 receiver cell type, direction in $A D$, and number of targeted adDEGs. j. The enriched biological pro572 cesses of targets shown in (i). 
573 Figure 6. APOE4-associated transcriptional differences and cognitive decline. a. The compari-

574 son of apoeDEGs and adDEGs for each cell type. The heatmaps show the number of apoeDEGs,

575 adDEGs and overlapping DEGs. The fold enrichment and FDR are shown in the barplot along with

576 heatmap. The significant ones are colored by blue or red. b. Enriched Gene Ontology biological pro-

577 cesses in E4 lower/higher genes in cEndo and Per1. The color for heatmap of term-gene pairs repre-

578 sents the effect size. The last three columns show enrichment/significance of each term and average

579 effect size of the genes. c. The average correlation of apoeDEGs with cognitive decline shown in the

580 heatmap. The bar plot shows the significance of lower correlation of E4 highly expressed genes with

581 cognitive decline. The boxplot (right) highlighted the most significant ones. $\mathbf{d}$. The number of decline-

582 higher genes (left) and decline-lower genes (right) in APOE4 and APOE3 individuals. The bar plot

583 shows the cell types with more cogDEGs in APOE4 individuals (>1.5 fold). e. The number and signifi-

584 cance of overlapping decline-higher/-lower genes between APOE4 and APOE3 individuals. f. Enriched

585 biological functions of APOE genotype specific/shared decline-higher/-lower genes in capillary endo-

586 thelial cells (top) and pericytes (bottom). 
Human brain tissues in this study were obtained from the Religious Orders Study and Rush Memory and Aging Project (ROSMAP, each approved by an Institutional Review Board (IRB) of Rush University Medical Center) with informed consent, an Anatomic Gift Act for organ donation, and a repository consent to allow the data to be shared ${ }^{114}$. Quantitative clinical and pathologic phenotypes of $A D$ were used to assess disease severity. These included global cognition proximate to death, and a measure of global AD pathology as well as the molecularly specific beta-amyloid and PHFtau tangles. Controls were defined as individuals with little to no AD pathology, whereas cases included a spectrum of AD pathology. Thus, case status was based solely based on AD pathology and other variables were allowed to freely associate, as previously reported ${ }^{115-119}$.

We isolated nuclei from frozen postmortem brain tissue as previously described ${ }^{120}$. Briefly, to avoid transcriptome changes due to protease processing, we homogenize the tissue in a Dounce grinder in the presence of low-concentration detergent to lyse the cell membrane and release intact nuclei. Lysates are filtered through 40 um cell strainers, and we purify nuclei using density-gradient centrifugation to eliminate cell debris. To prevent RNA degradation, we carry out all steps at $4^{\circ} \mathrm{C}$ and in the presence of RNase inhibitor (Takara). We use the isolated nuclei for the droplet-based 10x scRNA-seq assay, targeting 10k nuclei for each region of each individual and prepare libraries using Chromium Single-Cell 3' Reagent Kits v3 (10x Genomics, Pleasanton CA) according to the manufacturer's protocol. We sequenced pooled libraries using the NovaSeq 6000 S2 sequencing kits (100 cycles, Illumina).

\section{snRNA-seq data preprocessing}

609

We aligned the raw reads to human reference genome version GRCh38 (pre-mRNA) and quantified gene counts using CellRanger software v3.0.1 (10x Genomics, Pleasanton CA) ${ }^{121}$. The generated cellgene count matrix was processed using the Seurat R package v.4.0.3 $3^{122}$. We used a threshold of 500 unique molecular identifiers (UMls) to select cells, and a cut-off value of 50 cells to select genes for further analysis. We filtered out the cells with more than $10 \%$ mitochondrial genes. The gene count was normalized by the total counts for each cell, multiplied by 10000 , and then log-transformed. We identified the top 2000 highly variable genes for dimension reduction using Seurat default parameters. We used Harmony for batch correction ${ }^{123}$, and DoubletFinder to estimate doublet score with the parameter of $7.5 \%$ doublet formation rate ${ }^{124}$. The cells with high doublet scores ( 0.2 as cutoff) were discarded for further analysis. After generating clusters, the cluster that shows high expression of markers of two or more cell types was also treated as doublets and removed for further analysis.

621 For the full datasets with all cell types, we first annotated the cell type for each cluster based on the 
canonical markers of major cell types in the brain (including excitatory and inhibitory neuron, astrocyte, oligodendrocyte, OPC, microglia and vascular cell) ${ }^{120}$ and the enrichment of a large set of markers ${ }^{125}$

624 in highly expressed genes of each cluster. We next calculated the cell type score for each cell, which was represented by the average expression of a group of markers of each cell type ${ }^{125}$. The cells were selected as vascular cells for further integrative analysis only if (1) the clusters that the cells belong to were annotated as vascular cell types; (2) the cells show the specific high score of vascular cell types (highest score is 2 -fold higher than the second score). We had previously reported data from control individuals $^{12}$, and here report data from $A D$ individuals. regions for each cell type (expressed in at least $25 \%$ of endothelial cells and logarithm of fold change is higher than 0.25). For the comparison between AD and control, APOE3 and APOE4, cognitive decline, we applied MAST to measure the statistical significance for each gene based on a linear model ${ }^{126}$. The covariates including number of cells, number of expressed genes, age, sex, PMI, race, other dementia related pathology (Lewy body dementia, parkinson's disease and vascular contributions to cognitive impairment and dementia) were controlled in the model. The genes with FDR $<0.05$ and coefficient $>0.02$ were selected for further analysis. To confirm that these differences are biological and not statistical artifacts, we permuted the annotation of AD pathology for each individual and identified the adDEGs using the same computational pipeline, and found that the number of adDEGs

642 is significantly higher than expected by chance.

\section{RNA in situ hybridization}

644 For human postmortem samples, fresh frozen human PFC samples (BA region 9) were embedded in 645 Tissue-Tek OCT compound (Sakura, \#25608-930), cut at $10 \mu \mathrm{m}$ using a cryostat (Leica, CM3050S) 646 and collected on Superfrost Plus slides (Fischer Scientific, \#12-550-15). Tissue sections were stored 647 at $-80^{\circ} \mathrm{C}$ until further processing. RNAscope chromogenic $2.5 \mathrm{HD}$ duplex reagent kit (Advanced Cell 648 Diagnostic, \#322430) was used to perform RNA in situ hybridization according to the manufacturer's 649 instructions with the following modifications: tissue was fixed in 4\% paraformaldehyde for 30 minutes; 65030 minutes were allowed for C2 probe hybridization; overnight at room temperature was used for C1 651 probe hybridization; and xylene was not used prior to mounting. Probes used in this study include 652 CD31 (Advanced Cell Diagnostic, \#548451-C2, red) and INSR (Advanced Cell Diagnostic, \#406411, 653 green). Images were acquired using the brightfield settings of a Zeiss LSM 900 microscope.

655 Images were imported into QuPath (version 0.2.0-m8). Vessel segments were identified based on 656 CD31+ punctae, which formed visually distinct vessel-like segments. To quantify INSR expression 
657

658

659

660

661

662

663

664

665

666

667

668

669

670

671

672

673

674

675

676

677

678

679

680

681

682

683

684

685

686

687

688

689

690

691

692

within CD31+ cells, we manually counted the number of INSR+ punctae within each vascular segment. To calculate the density of INSR within each vascular segment, we quantified the ratio of INSR punctae within each vascular segment based on square micrometer surface area of each segment, then determined the frequency distribution for each density quantification between $A D$ and control patients.

\section{Prediction of regulators}

We predicted the upstream regulators of cell type markers and adDEGs using Enrichr in $\mathrm{R}$ based on three libraries including TRANSFAC and JASPAR, ChEA, and ENCODE TF ChIP-seq data ${ }^{127-129}$. We used adjusted $p$-value $<0.05$ as a cut-off to select the significant regulators. We kept the regulators with detected expression in the relevant cell types for further analysis. For the predicted upstream regulators of adDEGs, we tested the significance of shared targets between each pair of them in each geneset, and grouped these regulators into co-regulatory modules based on the hierarchical clustering of significance (-log10 of p-value by Fisher's exact test). We evaluated the regulators within one module by searching the protein-protein interaction network database STRING ${ }^{51}$ and downloaded the generated network as supporting information in this study. We then generated a matrix of zero-one to represent the regulatory relation between regulators and targets, performed hierarchical clustering to separate targets into distinct clusters and calculated the percentage of ones in the block (formed by gene cluster and regulator module) to determine if the target cluster was regulated by a regulator module (30\% as a cutoff). One target group could be regulated by zero, one or multiple regulator modules, and vice versa.

\section{Gene Ontology Enrichment Analysis}

We used Enrichr in $\mathrm{R}$ to perform enrichment analysis for Gene Ontology biological process (adjusted $p$-value $<0.05$ as a cut-off) ${ }^{130,131}$. The selected terms were manually combined according to the general functions including immune response, cell proliferation, cell migration, etc.

\section{Prediction of dynamic cell-cell communications in AD}

For each cell type (including vascular and non-vascular cell types in our brain datasets), we first clustered adDEGs into co-expression gene modules across all individuals, aggregating single-cell expression for each cell type within each individual. Then, for each pair of cell types, we generated the Pearson correlation coefficient matrix across all module pairs for that cell type pair, and estimated the significance using cor.test function in R. We used a value of adjusted p-value 0.05 as cut-off to select the significant correlated modules between two cell types. For each gene module, we performed Gene Ontology enrichment analysis to measure the importance of the module at the pathway level and removed the gene modules without enriched biological processes and signaling pathways. We combined four ligand-receptor databases (CellChatDB ${ }^{132}$, CellPhoneDB ${ }^{133}$, CellTalkDB ${ }^{134}$, and SingleCellSingalR ${ }^{135}$ ) to annotate the correlated modules as evidence for interacting cell-cell pairs mediated by ligand-receptor signaling pathways. We manually curated the selected ligand-receptor pairs in this 
693

694

695

696

697

698

699

700

701

702

703

704

705

706

707

708

709

710

711

712

713

714

715

study to remove the mislabeled or intracellular protein-protein interactions through literature searching and filtering. The final results include the interacting cell types, ligand, ligand-involved functions, receptor, receptor-involved functions, potential targets in signal receiver cell type, and direction of cell-cell communication in $A D$.

\section{adDEGs association with AD genetics}

We firstly downloaded AD-GWAS associated genes based on GWAS catalog annotation ${ }^{77}$ and summary statistics from Jansen et $\mathrm{al}^{73}$ using a subthreshold of $-\log 10$ ( $\mathrm{p}$-value) $>5$ to match genes from GWAS catalog annotation. The adDEGs in AD GWAS catalog were then mapped to AD variants based on the annotation in catalog. The variant with the lowest $p$-value for each gene was used to label position in the Manhattan plot. We used four types of linking evidence to evaluate the association between AD-variant and candidate genes: (a) physical chromatin conformation capture ( $\mathrm{Hi}-\mathrm{C}$ ) looping $^{78-81}$, (b) correlation-based enhancer-gene links ${ }^{82}$; (c) brain/heart/muscle-specific eQTLs at tissuelevel resolution ${ }^{83}$; and (d) vasculature cell-type specific single-cell eQTLs (sc-eQTLs) (in preparation). The association with at least two pieces of linking evidence were selected for visualization. The distance between variant and gene TSS and its rank among variant-associated genes were also calculated and visualized.

We also evaluated the association between $A D$-variant and predicted regulators of adDEGs using the four types of linking evidence mentioned above. We calculated the number of targets for each AD-associated regulator for each cell type and plotted the heatmap to show if the regulation is cell-type-specific or cell-type-shared. Similarly, we evaluated the association between AD-variant and ligands that meditates AD-related cell-cell communications using the four types of linking evidence and performed functional enrichment analysis for each target set to measure the functions of those targets as the downstream of $A D$-associated ligand in vascular cell types. 


\section{References}

1. Langen, U. H., Ayloo, S. \& Gu, C. Development and Cell Biology of the Blood-Brain Barrier. Annu. Rev. Cell Dev. Biol. 35, 591-613 (2019).

2. Banks, W. A. From blood-brain barrier to blood-brain interface: new opportunities for CNS drug delivery. Nat. Rev. Drug Discov. 15, 275-292 (2016).

3. Montagne, A., Zhao, Z. \& Zlokovic, B. V. Alzheimer's disease: A matter of blood-brain barrier dysfunction? J. Exp. Med. 214, 3151-3169 (2017).

4. Sweeney, M. D., Kisler, K., Montagne, A., Toga, A. W. \& Zlokovic, B. V. The role of brain vasculature in neurodegenerative disorders. Nat. Neurosci. 21, 1318-1331 (2018).

5. Varatharaj, A. \& Galea, I. The blood-brain barrier in systemic inflammation. Brain Behav. Immun. 60, 1-12 (2017).

6. Sweeney, M. D., Sagare, A. P. \& Zlokovic, B. V. Blood-brain barrier breakdown in Alzheimer disease and other neurodegenerative disorders. Nat. Rev. Neurol. 14, 133-150 (2018).

7. Zhao, Z., Nelson, A. R., Betsholtz, C. \& Zlokovic, B. V. Establishment and Dysfunction of the BloodBrain Barrier. Cell 163, 1064-1078 (2015).

8. Sweeney, M. D., Zhao, Z., Montagne, A., Nelson, A. R. \& Zlokovic, B. V. Blood-Brain Barrier: From Physiology to Disease and Back. Physiol. Rev. 99, 21-78 (2019).

9. Nelson, A. R., Sweeney, M. D., Sagare, A. P. \& Zlokovic, B. V. Neurovascular dysfunction and neurodegeneration in dementia and Alzheimer's disease. Biochim. Biophys. Acta 1862, 887-900 (2016).

10. Zlokovic, B. V. Neurovascular pathways to neurodegeneration in Alzheimer's disease and other disorders. Nat. Rev. Neurosci. 12, 723-738 (2011).

11. Vanlandewijck, M. et al. A molecular atlas of cell types and zonation in the brain vasculature. Nature 554, 475-480 (2018).

12. Garcia, F. J. et al. Single-cell dissection of the human cerebrovasculature in health and disease. doi:10.1101/2021.04.26.440975.

13. Yang, A. C. et al. A human brain vascular atlas reveals diverse cell mediators of Alzheimer's disease risk. doi:10.1101/2021.04.26.441262.

14. Wilhelm, I., Nyúl-Tóth, Á., Suciu, M., Hermenean, A. \& Krizbai, I. A. Heterogeneity of the blood-brain barrier. Tissue Barriers 4, e1143544 (2016).

15. Tran, K. A. et al. Endothelial $\beta$-Catenin Signaling Is Required for Maintaining Adult Blood-Brain Barrier Integrity and Central Nervous System Homeostasis. Circulation 133, 177-186 (2016).

16. Ganta, V. C. \& Annex, B. H. LMO2 (LIM Domain Only 2) and Endothelial Cell Migration in Developmental and Postnatal Angiogenesis. Arteriosclerosis, thrombosis, and vascular biology vol. 37 18061808 (2017).

17. Wei, G. et al. Ets1 and Ets2 are required for endothelial cell survival during embryonic angiogenesis. Blood 114, 1123-1130 (2009).

18. Slater, S. C. et al. MicroRNA-532-5p Regulates Pericyte Function by Targeting the Transcription Regulator BACH1 and Angiopoietin-1. Mol. Ther. 26, 2823-2837 (2018).

19. Xie, C. et al. Yap1 protein regulates vascular smooth muscle cell phenotypic switch by interaction with myocardin. J. Biol. Chem. 287, 14598-14605 (2012).

20. Shen, T. et al. YAP1 plays a key role of the conversion of normal fibroblasts into cancer-associated fibroblasts that contribute to prostate cancer progression. J. Exp. Clin. Cancer Res. 39, 1-16 (2020).

21. Park, R. et al. Yap is required for ependymal integrity and is suppressed in LPA-induced hydrocephalus. Nat. Commun. 7, 10329 (2016).

22. Wegiel, J. et al. Vascular fibrosis and calcification in the hippocampus in aging, Alzheimer disease, and Down syndrome. Acta Neuropathol. 103, 333-343 (2002).

23. Rauramaa, T. et al. Consensus recommendations on pathologic changes in the hippocampus: a postmortem multicenter inter-rater study. J. Neuropathol. Exp. Neurol. 72, 452-461 (2013).

24. de Brouwer, E. J. M. et al. Hippocampal Calcifications: Risk Factors and Association with Cognitive Function. Radiology 288, 815-820 (2018).

25. Peters, M. E. M. et al. Histological validation of calcifications in the human hippocampus as seen on computed tomography. PLoS One 13, e0197073 (2018).

26. Hawkes, C. A. et al. Regional differences in the morphological and functional effects of aging on cerebral basement membranes and perivascular drainage of amyloid- $\beta$ from the mouse brain. Aging 
Cell 12, 224-236 (2013).

27. Mestre, H. et al. Cerebrospinal fluid influx drives acute ischemic tissue swelling. Science 367, (2020).

28. Zhang, $X$. et al. High-resolution mapping of brain vasculature and its impairment in the hippocampus of Alzheimer's disease mice. Natl Sci Rev 6, 1223-1238 (2019).

29. Montagne, A. et al. Blood-brain barrier breakdown in the aging human hippocampus. Neuron $\mathbf{8 5}$, 296-302 (2015).

30. Deo, A. K. et al. Activity of P-Glycoprotein, a $\beta$-Amyloid Transporter at the Blood-Brain Barrier, Is Compromised in Patients with Mild Alzheimer Disease. J. Nucl. Med. 55, 1106-1111 (2014).

31. van Assema, D. M. E. et al. Blood-brain barrier P-glycoprotein function in Alzheimer's disease. Brain 135, 181-189 (2012).

32. Patching, S. G. Glucose Transporters at the Blood-Brain Barrier: Function, Regulation and Gateways for Drug Delivery. Mol. Neurobiol. 54, 1046-1077 (2017).

33. Gejl, M. et al. Blood-Brain Glucose Transfer in Alzheimer's disease: Effect of GLP-1 Analog Treatment. Scientific Reports vol. 7 (2017).

34. Hamilton, N. B., Attwell, D. \& Hall, C. N. Pericyte-mediated regulation of capillary diameter: a component of neurovascular coupling in health and disease. Front. Neuroenergetics 2, (2010).

35. Nortley, R. et al. Amyloid $\beta$ oligomers constrict human capillaries in Alzheimer's disease via signaling to pericytes. Science 365, (2019).

36. Cho, S.-J. et al. Altered expression of Notch1 in Alzheimer's disease. PLoS One 14, e0224941 (2019).

37. Vagnucci, A. H., Jr \& Li, W. W. Alzheimer's disease and angiogenesis. Lancet 361, 605-608 (2003).

38. Vromman, A. et al. $\beta$-Amyloid context intensifies vascular smooth muscle cells induced inflammatory response and de-differentiation. Aging Cell 12, 358-369 (2013).

39. Spinelli, M., Fusco, S. \& Grassi, C. Brain Insulin Resistance and Hippocampal Plasticity: Mechanisms and Biomarkers of Cognitive Decline. Front. Neurosci. 13, 788 (2019).

40. Spencer, B., Rank, L., Metcalf, J. \& Desplats, P. Identification of Insulin Receptor Splice Variant B in Neurons by in situ Detection in Human Brain Samples. Sci. Rep. 8, 4070 (2018).

41. Kawasawa, Y. I. et al. RNA-seq analysis of developing olfactory bulb projection neurons. Mol. Cell. Neurosci. 74, 78-86 (2016).

42. Chong, A. C. N., Vogt, M. C., Hill, A. S., Brüning, J. C. \& Zeltser, L. M. Central insulin signaling modulates hypothalamus-pituitary-adrenal axis responsiveness. Molecular Metabolism vol. 4 83-92 (2015).

43. Arvanitakis, Z. et al. Brain insulin signaling and cerebrovascular disease in human postmortem brain. Acta Neuropathol Commun 9, 71 (2021).

44. Arvanitakis, Z. et al. Brain Insulin Signaling, Alzheimer Disease Pathology, and Cognitive Function. Ann. Neurol. 88, 513-525 (2020).

45. Talbot, K. et al. Demonstrated brain insulin resistance in Alzheimer's disease patients is associated with IGF-1 resistance, IRS-1 dysregulation, and cognitive decline. J. Clin. Invest. 122, 1316-1338 (2012).

46. van Heemst, D. Insulin, IGF-1 and longevity. Aging Dis. 1, 147-157 (2010).

47. Bhatia, S., Kim, W. S., Shepherd, C. E. \& Halliday, G. M. Apolipoprotein D Upregulation in Alzheimer's Disease but Not Frontotemporal Dementia. J. Mol. Neurosci. 67, 125-132 (2019).

48. Dassati, S., Waldner, A. \& Schweigreiter, R. Apolipoprotein D takes center stage in the stress response of the aging and degenerative brain. Neurobiol. Aging 35, (2014).

49. Lau, S.-F., Cao, H., Fu, A. K. Y. \& Ip, N. Y. Single-nucleus transcriptome analysis reveals dysregulation of angiogenic endothelial cells and neuroprotective glia in Alzheimer's disease. Proc. Natl. Acad. Sci. U. S. A. 117, 25800-25809 (2020).

50. Leng, K. et al. Molecular characterization of selectively vulnerable neurons in Alzheimer's disease. Nat. Neurosci. 24, 276-287 (2021).

51. Szklarczyk, D. et al. The STRING database in 2021: customizable protein-protein networks, and functional characterization of user-uploaded gene/measurement sets. Nucleic Acids Res. 49, D605D612 (2021).

52. Kim, K.-J. et al. STAT3 activation in endothelial cells is important for tumor metastasis via increased cell adhesion molecule expression. Oncogene 36, 5445-5459 (2017). 
53. Wei, D. et al. Stat3 activation regulates the expression of vascular endothelial growth factor and human pancreatic cancer angiogenesis and metastasis. Oncogene 22, 319-329 (2003).

54. Zhang, B. et al. A dynamic H3K27ac signature identifies VEGFA-stimulated endothelial enhancers and requires EP300 activity. Genome Res. 23, 917-927 (2013).

55. Marconcini, L. et al. c-fos-induced growth factor/vascular endothelial growth factor D induces angiogenesis in vivo and in vitro. Proc. Natl. Acad. Sci. U. S. A. 96, 9671-9676 (1999).

56. Battle, T. E., Lynch, R. A. \& Frank, D. A. Signal transducer and activator of transcription 1 activation in endothelial cells is a negative regulator of angiogenesis. Cancer Res. 66, 3649-3657 (2006).

57. Paneni, F. et al. Deletion of the activated protein-1 transcription factor JunD induces oxidative stress and accelerates age-related endothelial dysfunction. Circulation 127, 1229-40, e1-21 (2013).

58. Wang, N. et al. c-Jun triggers apoptosis in human vascular endothelial cells. Circ. Res. 85, 387-393 (1999).

59. Mahamud, M. R. et al. GATA2 controls lymphatic endothelial cell junctional integrity and lymphovenous valve morphogenesis through. Development 146, (2019).

60. Chen, M. B. et al. Brain Endothelial Cells Are Exquisite Sensors of Age-Related Circulatory Cues. Cell Rep. 30, 4418-4432.e4 (2020).

61. Hallock, P. \& Thomas, M. A. Integrating the Alzheimer's disease proteome and transcriptome: a comprehensive network model of a complex disease. OMICS 16, 37-49 (2012).

62. Zhao, L. et al. Pharmacologically reversible zonation-dependent endothelial cell transcriptomic changes with neurodegenerative disease associations in the aged brain. Nat. Commun. 11, 4413 (2020).

63. Baron, C. S. et al. Single-cell transcriptomics reveal the dynamic of haematopoietic stem cell production in the aorta. Nat. Commun. 9, 2517 (2018).

64. Ohnesorge, N. et al. Erk5 activation elicits a vasoprotective endothelial phenotype via induction of Kruppel-like factor 4 (KLF4). J. Biol. Chem. 285, 26199-26210 (2010).

65. Pugacheva, E. M. et al. CTCF mediates chromatin looping via N-terminal domain-dependent cohesin retention. Proc. Natl. Acad. Sci. U. S. A. 117, 2020-2031 (2020).

66. Xu, L., Nirwane, A. \& Yao, Y. Basement membrane and blood-brain barrier. Stroke Vasc Neurol 4, 78-82 (2019).

67. Javier-Torrent, M. et al. Presenilin/Y-secretase-dependent EphA3 processing mediates axon elongation through non-muscle myosin IIA. Elife 8, (2019).

68. McMillin, M. A. et al. TGF $\beta 1$ exacerbates blood-brain barrier permeability in a mouse model of hepatic encephalopathy via upregulation of MMP9 and downregulation of claudin-5. Lab. Invest. 95, 903-913 (2015).

69. Rustenhoven, J. et al. TGF-beta1 regulates human brain pericyte inflammatory processes involved in neurovasculature function. J. Neuroinflammation 13, 37 (2016).

70. von Bernhardi, R., Cornejo, F., Parada, G. E. \& Eugenín, J. Role of TGF $\beta$ signaling in the pathogenesis of Alzheimer's disease. Front. Cell. Neurosci. 9, 426 (2015).

71. Kashima, R. \& Hata, A. The role of TGF- $\beta$ superfamily signaling in neurological disorders. Acta Biochim. Biophys. Sin. 50, 106-120 (2018).

72. van Cruijsen, H., Giaccone, G. \& Hoekman, K. Epidermal growth factor receptor and angiogenesis: Opportunities for combined anticancer strategies. Int. J. Cancer 117, 883-888 (2005).

73. Jansen, I. E. et al. Genome-wide meta-analysis identifies new loci and functional pathways influencing Alzheimer's disease risk. Nat. Genet. 51, 404-413 (2019).

74. Kunkle, B. W. et al. Genetic meta-analysis of diagnosed Alzheimer's disease identifies new risk loci and implicates $A \beta$, tau, immunity and lipid processing. Nat. Genet. 51, 414-430 (2019).

75. Kang, S. et al. Potential Novel Genes for Late-Onset Alzheimer's Disease in East-Asian Descent Identified by APOE-Stratified Genome-Wide Association Study. J. Alzheimers. Dis. 82, 1451-1460 (2021).

76. Pallejà, A., Horn, H., Eliasson, S. \& Jensen, L. J. DistiLD Database: diseases and traits in linkage disequilibrium blocks. Nucleic Acids Res. 40, D1036-40 (2012).

77. Buniello, A. et al. The NHGRI-EBI GWAS Catalog of published genome-wide association studies, targeted arrays and summary statistics 2019. Nucleic Acids Res. 47, D1005-D1012 (2019).

78. Kaul, A., Bhattacharyya, S. \& Ay, F. Identifying statistically significant chromatin contacts from Hi-C data with FitHiC2. Nat. Protoc. 15, 991-1012 (2020). 
79. Lalonde, S. et al. Integrative analysis of vascular endothelial cell genomic features identifies AIDA as a coronary artery disease candidate gene. Genome Biol. 20, 133 (2019).

80. Niskanen, $\mathrm{H}$. et al. Endothelial cell differentiation is encompassed by changes in long range interactions between inactive chromatin regions. Nucleic Acids Res. 46, 1724-1740 (2018).

81. Mumbach, M. R. et al. Enhancer connectome in primary human cells identifies target genes of disease-associated DNA elements. Nat. Genet. 49, 1602-1612 (2017).

82. Boix, C. A., James, B. T., Park, Y. P., Meuleman, W. \& Kellis, M. Regulatory genomic circuitry of human disease loci by integrative epigenomics. Nature 590, 300-307 (2021).

83. GTEx Consortium. The GTEx Consortium atlas of genetic regulatory effects across human tissues. Science 369, 1318-1330 (2020).

84. Chang, T.-Y., Yamauchi, Y., Hasan, M. T. \& Chang, C. Cellular cholesterol homeostasis and Alzheimer's disease. J. Lipid Res. 58, 2239-2254 (2017).

85. Sun, Y. et al. Nuclear receptor RORa regulates pathologic retinal angiogenesis by modulating SOCS3-dependent inflammation. Proc. Natl. Acad. Sci. U. S. A. 112, 10401-10406 (2015).

86. Saint-Pol, J. et al. Brain pericytes ABCA1 expression mediates cholesterol efflux but not cellular amyloid- $\beta$ peptide accumulation. J. Alzheimers. Dis. 30, 489-503 (2012).

87. Koldamova, R., Fitz, N. F. \& Lefterov, I. The role of ATP-binding cassette transporter A1 in Alzheimer's disease and neurodegeneration. Biochim. Biophys. Acta 1801, 824-830 (2010).

88. Koldamova, R. P. et al. The liver $X$ receptor ligand T0901317 decreases amyloid beta production in vitro and in a mouse model of Alzheimer's disease. J. Biol. Chem. 280, 4079-4088 (2005).

89. Robert, J., Osto, E. \& von Eckardstein, A. The Endothelium Is Both a Target and a Barrier of HDL's Protective Functions. Cells 10, (2021).

90. Husemann, J. \& Silverstein, S. C. Expression of scavenger receptor class B, type I, by astrocytes and vascular smooth muscle cells in normal adult mouse and human brain and in Alzheimer's disease brain. Am. J. Pathol. 158, 825-832 (2001).

91. Rothaug, M., Becker-Pauly, C. \& Rose-John, S. The role of interleukin-6 signaling in nervous tissue. Biochim. Biophys. Acta 1863, 1218-1227 (2016).

92. Choi, J. M., Rotimi, O. O., O'Carroll, S. J. \& Nicholson, L. F. B. IL-6 stimulates a concentration-dependent increase in MCP-1 in immortalised human brain endothelial cells. F1000Res. 5, 270 (2016).

93. Zhang, L., Ju, X., Cheng, Y., Guo, X. \& Wen, T. Identifying Tmem59 related gene regulatory network of mouse neural stem cell from a compendium of expression profiles. BMC Syst. Biol. 5, 152 (2011).

94. Kong, W., Mou, X., Zhi, X., Zhang, X. \& Yang, Y. Dynamic regulatory network reconstruction for Alzheimer's disease based on matrix decomposition techniques. Comput. Math. Methods Med. 2014, 891761 (2014).

95. Waselle, L. et al. Involvement of the Rab27 binding protein Slac2c/MyRIP in insulin exocytosis. Mol. Biol. Cell 14, 4103-4113 (2003).

96. Sörgjerd, K. M. et al. Human prefoldin inhibits amyloid- $\beta(A \beta)$ fibrillation and contributes to formation of nontoxic A $\beta$ aggregates. Biochemistry 52, 3532-3542 (2013).

97. Liang, J. et al. The functions and mechanisms of prefoldin complex and prefoldin-subunits. Cell Biosci. 10, 87 (2020).

98. Zhou, Y. et al. TCF7L2 is a master regulator of insulin production and processing. Hum. Mol. Genet. 23, 6419-6431 (2014).

99. Facchinello, N. et al. Tcf7l2 plays pleiotropic roles in the control of glucose homeostasis, pancreas morphology, vascularization and regeneration. Scientific Reports vol. 7 (2017).

100. Srivastava, R. et al. TCF7L2 (Transcription Factor 7-Like 2) Regulation of GATA6 (GATA-Binding Protein 6)-Dependent and -Independent Vascular Smooth Muscle Cell Plasticity and Intimal Hyperplasia. Arterioscler. Thromb. Vasc. Biol. 39, 250-262 (2019).

101.Marcello, E., Borroni, B., Pelucchi, S., Gardoni, F. \& Di Luca, M. ADAM10 as a therapeutic target for brain diseases: from developmental disorders to Alzheimer's disease. Expert Opin. Ther. Targets 21, 1017-1026 (2017).

102. Garbers, C. et al. Species specificity of ADAM10 and ADAM17 proteins in interleukin-6 (IL-6) transsignaling and novel role of ADAM10 in inducible IL-6 receptor shedding. J. Biol. Chem. 286, 1480414811 (2011).

103. Groot, A. J. \& Vooijs, M. A. The role of Adams in Notch signaling. Adv. Exp. Med. Biol. 727, 15-36 (2012). 
104. Matthews, A. L. et al. Regulation of Leukocytes by TspanC8 Tetraspanins and the 'Molecular Scissor' ADAM10. Front. Immunol. 9, 1451 (2018).

105. Go, G.-W. \& Mani, A. Low-density lipoprotein receptor (LDLR) family orchestrates cholesterol homeostasis. Yale J. Biol. Med. 85, 19-28 (2012).

106. Yamazaki, Y., Zhao, N., Caulfield, T. R., Liu, C.-C. \& Bu, G. Apolipoprotein E and Alzheimer disease: pathobiology and targeting strategies. Nat. Rev. Neurol. 15, 501-518 (2019).

107.Ridge, P. G., Mukherjee, S., Crane, P. K., Kauwe, J. S. K. \& Alzheimer's Disease Genetics Consortium. Alzheimer's disease: analyzing the missing heritability. PLoS One 8, e79771 (2013).

108. Blanchard, J. W. et al. Reconstruction of the human blood-brain barrier in vitro reveals a pathogenic mechanism of APOE4 in pericytes. Nat. Med. 26, 952-963 (2020).

109. Hultman, K., Strickland, S. \& Norris, E. H. The APOE $\varepsilon 4 / \varepsilon 4$ genotype potentiates vascular fibrin(ogen) deposition in amyloid-laden vessels in the brains of Alzheimer's disease patients. $J$. Cereb. Blood Flow Metab. 33, 1251-1258 (2013).

110. Halliday, M. R. et al. Accelerated pericyte degeneration and blood-brain barrier breakdown in apolipoprotein E4 carriers with Alzheimer's disease. J. Cereb. Blood Flow Metab. 36, 216-227 (2016).

111. Montagne, A. et al. APOE4 leads to blood-brain barrier dysfunction predicting cognitive decline. Nature 581, 71-76 (2020).

112. Thambisetty, M., Beason-Held, L., An, Y., Kraut, M. A. \& Resnick, S. M. APOE epsilon4 genotype and longitudinal changes in cerebral blood flow in normal aging. Arch. Neurol. 67, 93-98 (2010).

113. Leaston, J. et al. Neurovascular imaging with QUTE-CE MRI in APOE4 rats reveals early vascular abnormalities. PLoS One 16, e0256749 (2021).

114.Bennett, D. A. et al. Religious Orders Study and Rush Memory and Aging Project. J. Alzheimers. Dis. 64, S161-S189 (2018).

115. Bennett, D. A. et al. Natural history of mild cognitive impairment in older persons. Neurology 59, 198-205 (2002).

116. Bennett, D. A. et al. Apolipoprotein E epsilon4 allele, AD pathology, and the clinical expression of Alzheimer's disease. Neurology 60, 246-252 (2003).

117. Bennett, D. A. et al. Neuropathology of older persons without cognitive impairment from two community-based studies. Neurology 66, 1837-1844 (2006).

118. Bennett, D. A., Schneider, J. A., Wilson, R. S., Bienias, J. L. \& Arnold, S. E. Neurofibrillary tangles mediate the association of amyloid load with clinical Alzheimer disease and level of cognitive function. Arch. Neurol. 61, 378-384 (2004).

119. Bennett, D. A. et al. Decision rules guiding the clinical diagnosis of Alzheimer's disease in two community-based cohort studies compared to standard practice in a clinic-based cohort study. Neuroepidemiology 27, 169-176 (2006).

120. Mathys, H. et al. Single-cell transcriptomic analysis of Alzheimer's disease. Nature 570, 332-337 (2019).

121.Zheng, G. X. Y. et al. Massively parallel digital transcriptional profiling of single cells. Nat. Commun. 8, 14049 (2017).

122. Hao, Y. et al. Integrated analysis of multimodal single-cell data. Cell 184, 3573-3587.e29 (2021).

123. Korsunsky, I. et al. Fast, sensitive and accurate integration of single-cell data with Harmony. Nat. Methods 16, 1289-1296 (2019).

124. McGinnis, C. S., Murrow, L. M. \& Gartner, Z. J. DoubletFinder: Doublet Detection in Single-Cell RNA Sequencing Data Using Artificial Nearest Neighbors. Cell Syst 8, 329-337.e4 (2019).

125. Wang, D. et al. Comprehensive functional genomic resource and integrative model for the human brain. Science 362, (2018).

126. Finak, G. et al. MAST: a flexible statistical framework for assessing transcriptional changes and characterizing heterogeneity in single-cell RNA sequencing data. Genome Biol. 16, 278 (2015).

127. Chen, E. Y. et al. Enrichr: interactive and collaborative HTML5 gene list enrichment analysis tool. BMC Bioinformatics 14, 128 (2013).

128. Xie, Z. et al. Gene Set Knowledge Discovery with Enrichr. Curr Protoc 1, e90 (2021).

129. Kuleshov, M. V. et al. Enrichr: a comprehensive gene set enrichment analysis web server 2016 update. Nucleic Acids Res. 44, W90-7 (2016).

130. Ashburner, M. et al. Gene Ontology: tool for the unification of biology. Nat. Genet. 25, 25-29 (2000). 
131. The Gene Ontology Consortium. The Gene Ontology Resource: 20 years and still GOing strong. Nucleic Acids Res. 47, D330-D338 (2019).

132. Jin, S. et al. Inference and analysis of cell-cell communication using CellChat. Nat. Commun. 12, 120 (2021).

133. Efremova, M., Vento-Tormo, M., Teichmann, S. A. \& Vento-Tormo, R. CellPhoneDB: inferring cellcell communication from combined expression of multi-subunit ligand-receptor complexes. Nat. Protoc. 15, 1484-1506 (2020).

134. Shao, X. et al. CellTalkDB: a manually curated database of ligand-receptor interactions in humans and mice. Brief. Bioinform. 22, (2021).

135. Cabello-Aguilar, S. et al. SingleCellSignalR: inference of intercellular networks from single-cell transcriptomics. Nucleic Acids Res. 48, e55-e55 (2020).

\section{Acknowledgements}

We thank the study participants and staff of the Rush Alzheimer's Disease Center. This work was supported in part by NIH grants AG054012, AG058002, AG062377, NS110453, NS115064, AG062335, NS127187 (M.K., L.-H.T.); AG067151, MH109978, MH119509, HG008155, DA053631 (M.K.); P30AG10161, P30AG72975, R01AG15819, R01AG17917, U01AG46152, U01AG61356, and R01AG57473 (D.A.B.); and the Cure Alzheimer's Foundation CIRCUITS consortium (M.K., L.-H.T); The JPB Foundation (L.-H.T.); Robert A. and Renee Belfer (L.-H.T.); N.S. was supported by Takeda Fellowship from Takeda Pharmaceutical Company. We thank Carles A. Boix, Lei Hou and Patricia Purcell for scientific suggestions.

\section{Code Availability}

Code used in this study is available upon reasonable request from the corresponding authors.

\section{Data Availability}

Count matrices for all cells analyzed in this study are uploaded with this submission as Supplementary Data and at http://compbio.mit.edu/scADbbb/. Interactive website is linked from http://compbio.mit.edu/scADbbb/. ROSMAP data can be requested at https://www.radc.rush.edu.

\section{Author Contributions}

N.S., M.K., and L.-H.T. conceived and designed the study; M.K. and L.-H.T. supervised the study; N.S. developed the computational framework and conducted data analysis with assistance from Y.P..; H.M., K.G., X.J. and A.P.N performed snRNA-seq profiling. L.A. and M.H.M. performed in situ hybridization and quantification with help from A.B.; D.A.B. provided post mortem samples and scientific input; and N.S. and M.K. wrote the paper with comments from all authors. 


\section{Extended Data Figures}

a

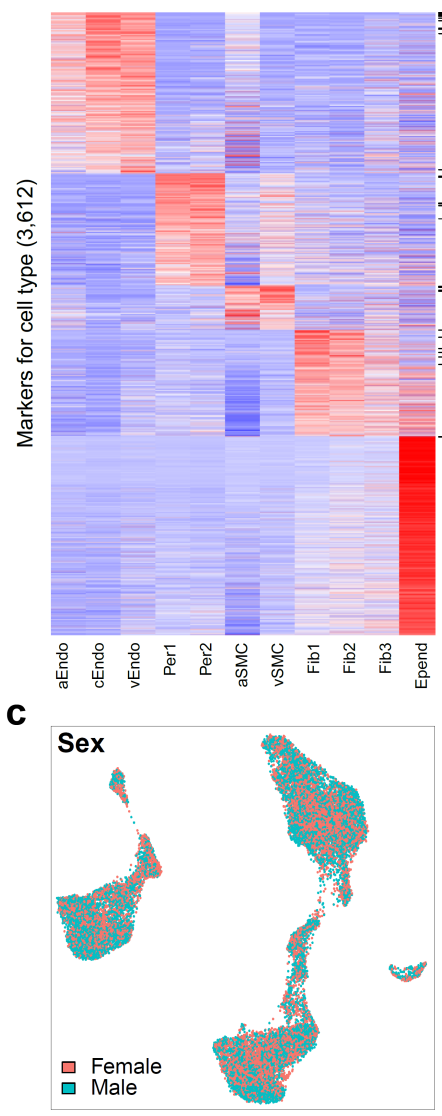

f

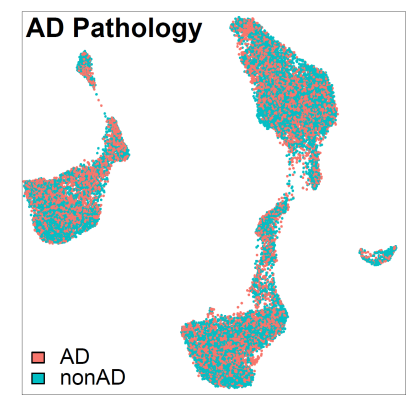

i

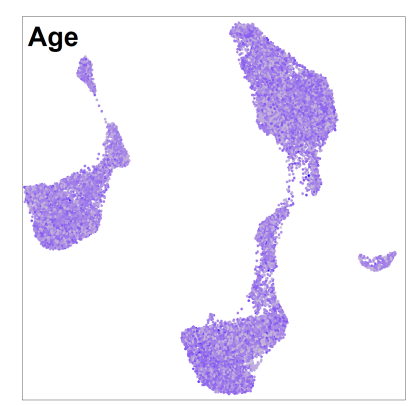

b

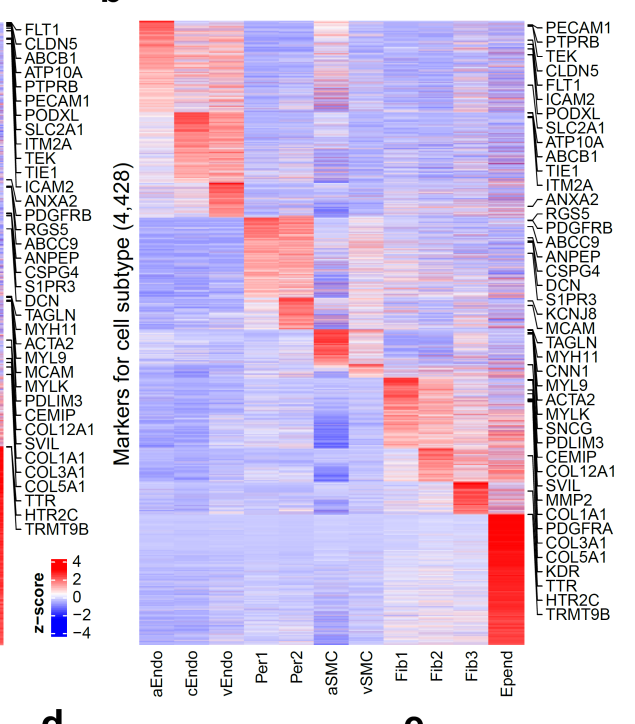

d

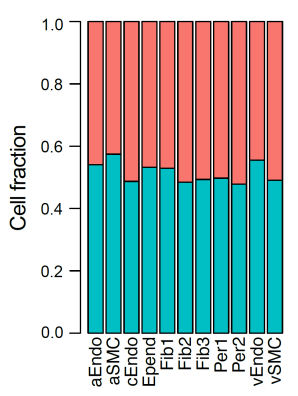

g

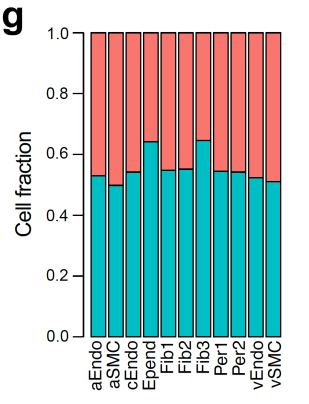

j

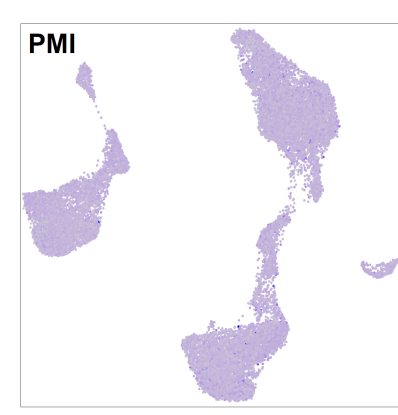

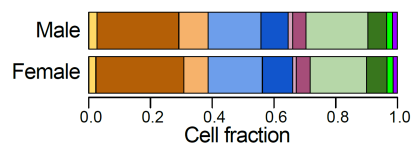

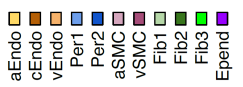

h

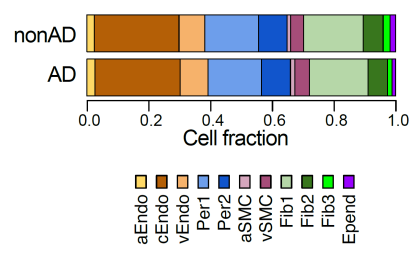

Extended Data Figure 1. Brain Vasculature Characterization across Six Brain Regions. a-b. Markers for vascular cell types (a) and cell subtypes (b). c-e. Cerebrovascular cell distribution by sex. UMAP of brain vascular nuclei labeled by sex (c), cell fraction across sex for each cell type (d), and cell fraction across cell types for male and female individuals (e). $\mathbf{f}-\mathbf{h}$. Cerebrovascular cell distribution by AD diagnosis. UMAP of brain vascular nuclei labeled by AD diagnosis $(\mathbf{f})$, cell fraction across AD diagnosis for each cell type (g), and cell fraction across cell types for AD and control individuals (h). i-j. UMAP of vascular nuclei with age (i) and PMI (j). k. Heatmap to show the number of highly expressed brDEGs of each region for each cell type. 
bioRxiv preprint doi: https://doi.org/10.1101/2022.02.09.479797; this version posted February 10,2022 . The copyright holder for this preprint (which was not certified by peer review) is the author/funder. All rights reserved. No reuse allowed without permission.
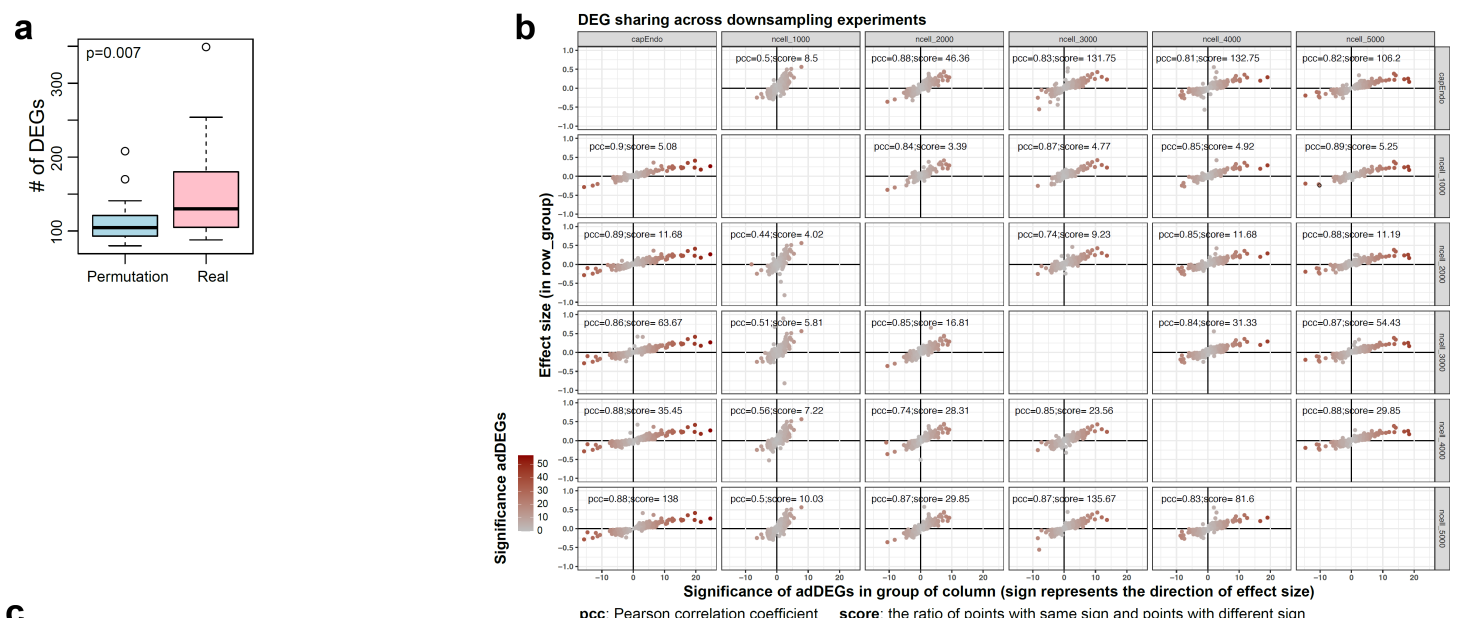

C Significance of adDEGs in group of column (sign represents the direction of effect sizen
pcc: Pearson correlation coefficient $\quad$ score: the ratio of points with same sign and points with different sign
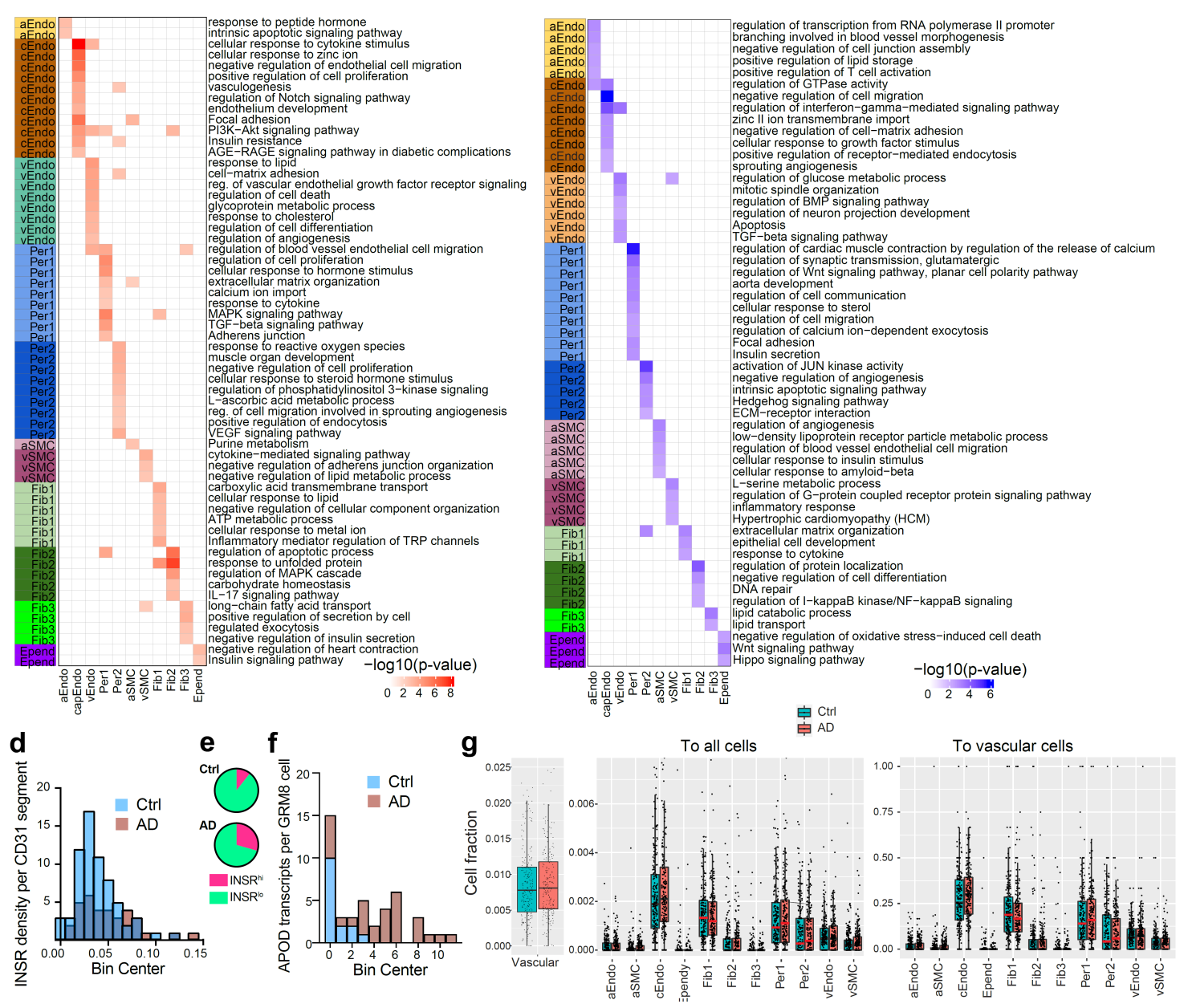

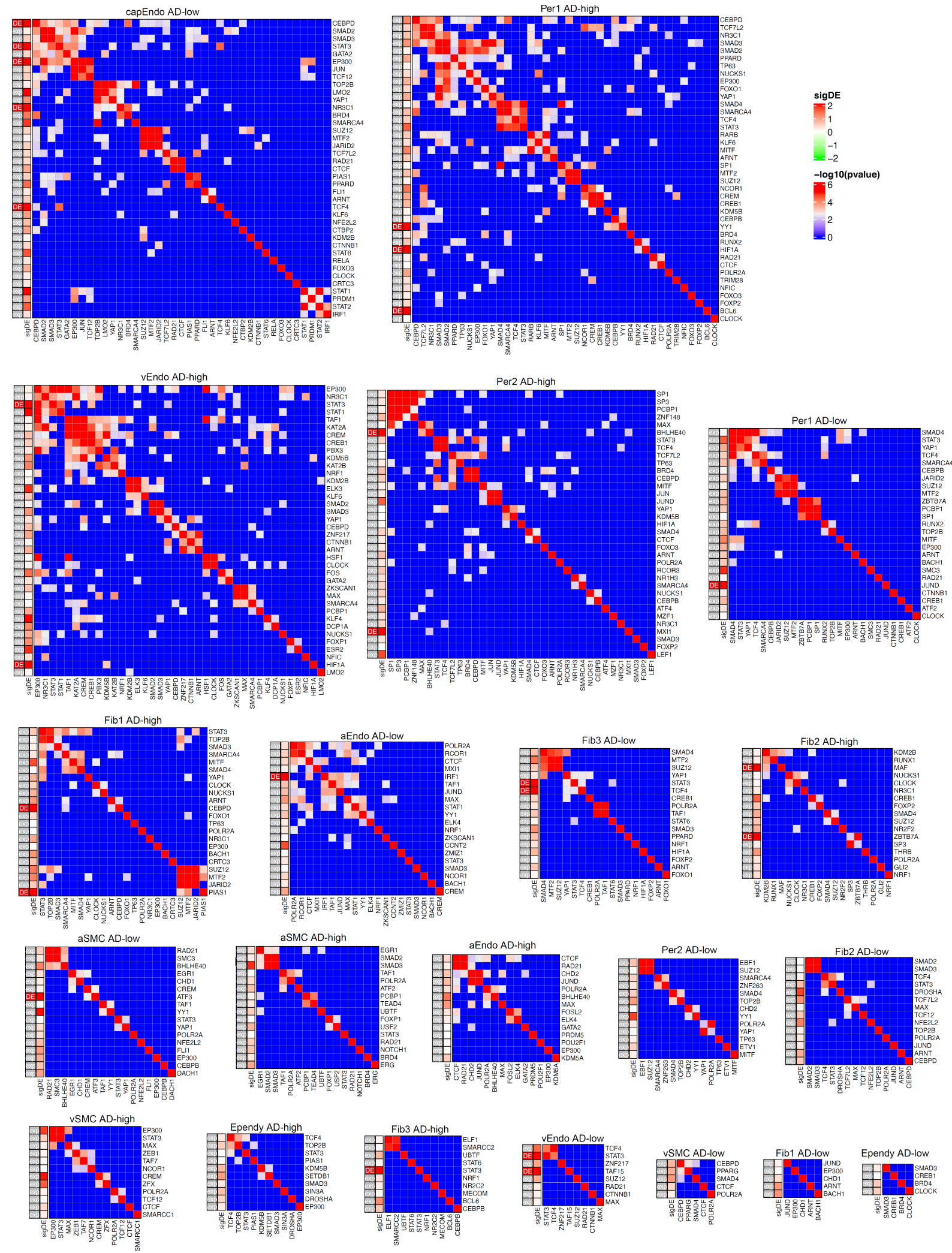

Extended Data Figure 3. Upstream regulators of adDEGs. Regulator modules of adDEGs in 11 cell types. For each heatmap, the first column on the left shows if the regulator is significantly differentially expressed. The second column shows the level of differential significance represented by $-\log 10(p-$ value). 


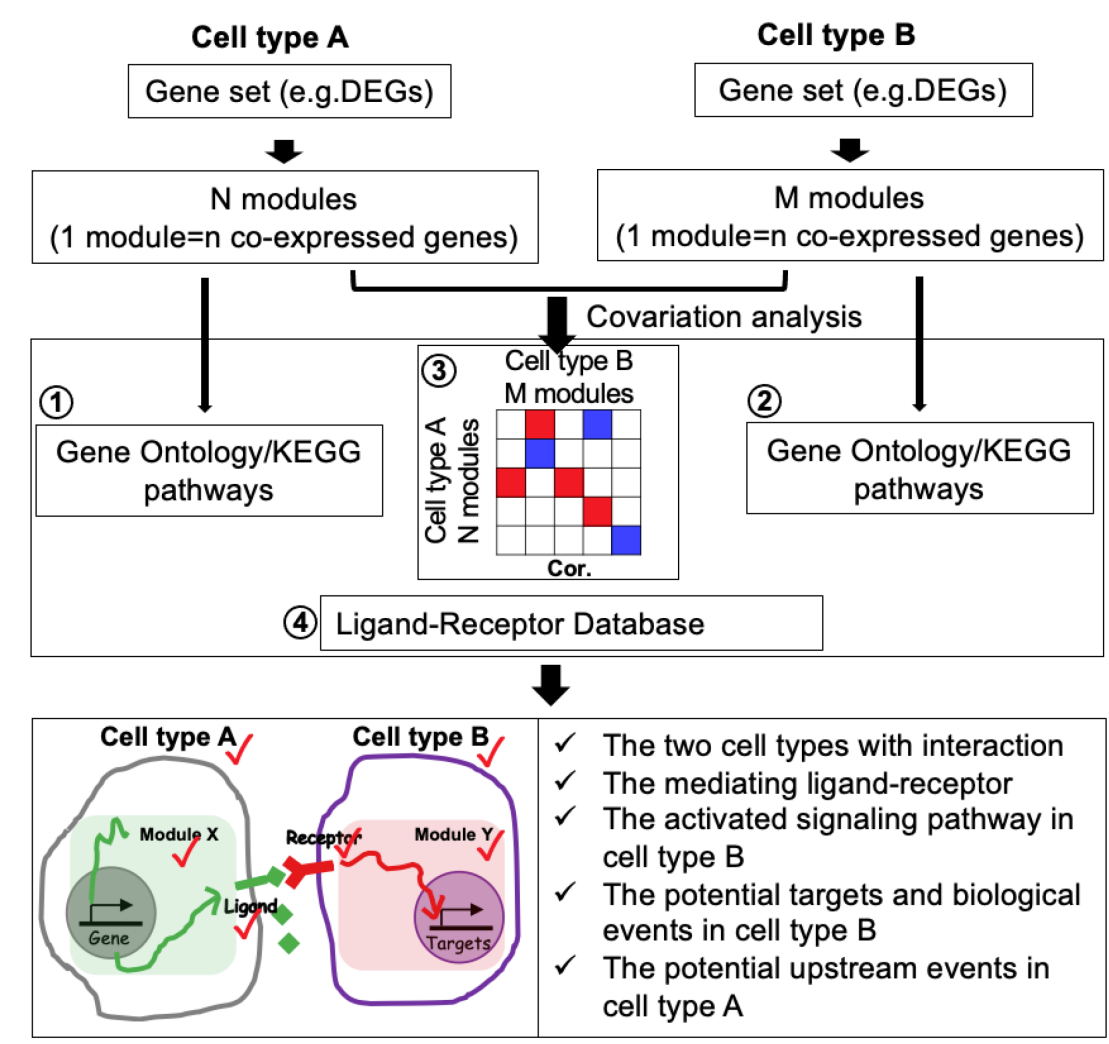

b

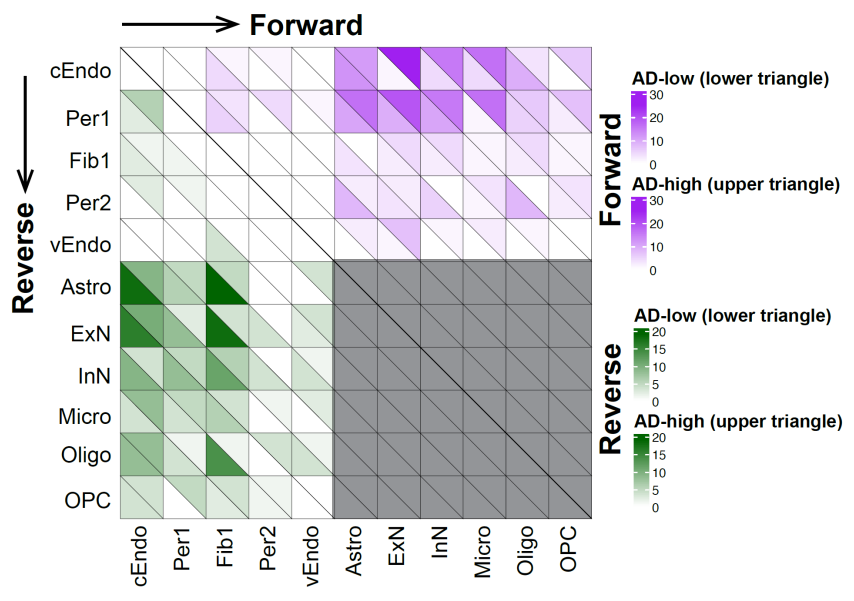

C

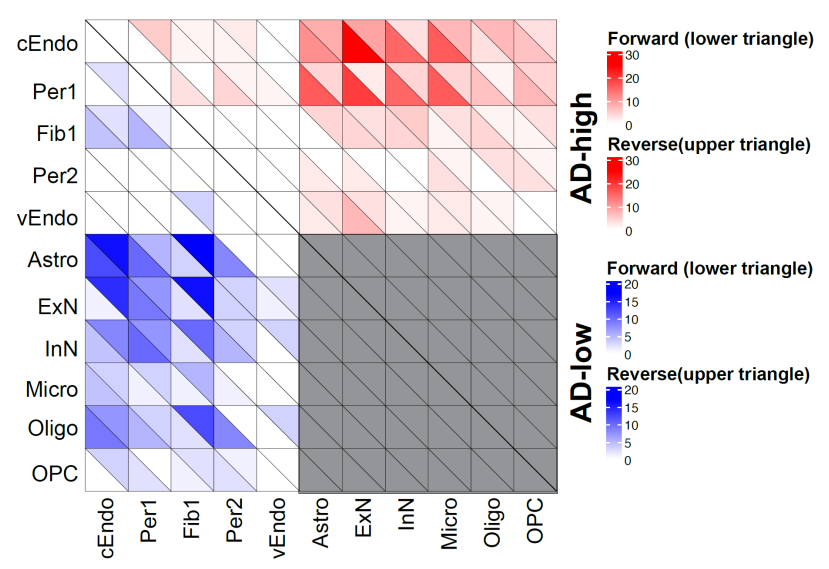

Extended Data Figure 4. Dynamics of cell-cell communications between vascular cell types and neuron/glial cells in AD. a. Computational framework to infer cell-cell communications. For each cell type, a set of genes were clustered into a number of co-expressed modules. The pairwise Pearson correlation coefficient was calculated between modules from each pair of cell types. The significant correlated modules, functional enrichment and ligand-receptor pairs were integrated into the prediction of cell-cell communication. The output includes the interacting cell types, ligand, ligand-involved functions, receptor, receptor-involved functions, potential targets in signal receiver cell type, and direction of cell-cell communication in $\mathrm{AD}$. b. The numbers of AD-lower (lower triangle of each square) and AD-higher (upper triangle of each square) in forward interactions from row to column (for example, the first row means the interaction from cEndo to other cell types) (upper triangle of the heatmap) and reverse interactions from column to row (for example, the first column means the interaction from other cell types to cEndo) (lower triangle of the heatmap). c. The numbers of forward (lower triangle of each square) and reverse (upper triangle of each square) in AD-higher interactions (upper triangle of the heatmap) and AD-lower interactions (lower triangle of the heatmap). 
a

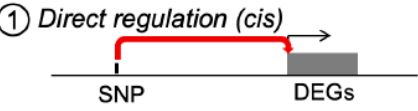

(2) Indirect regulation (trans)

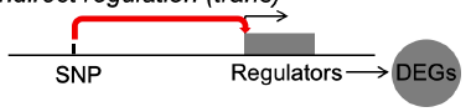

(3)Indirect regulation (signaling)

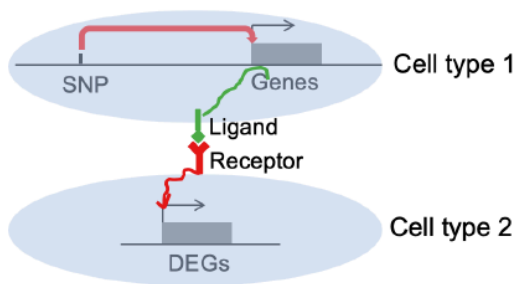

b

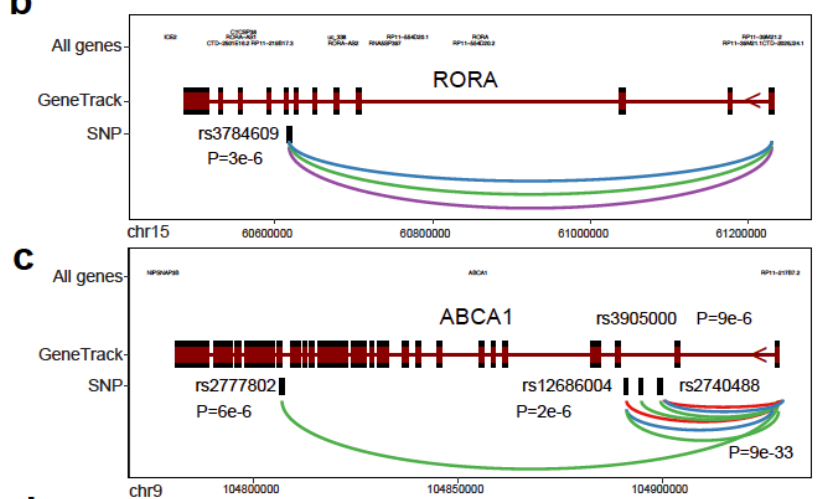

d
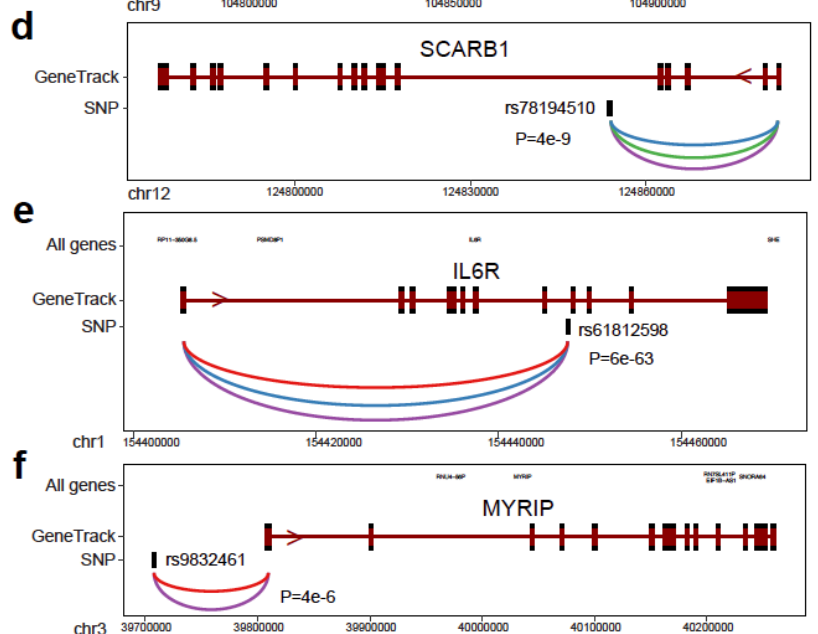

g
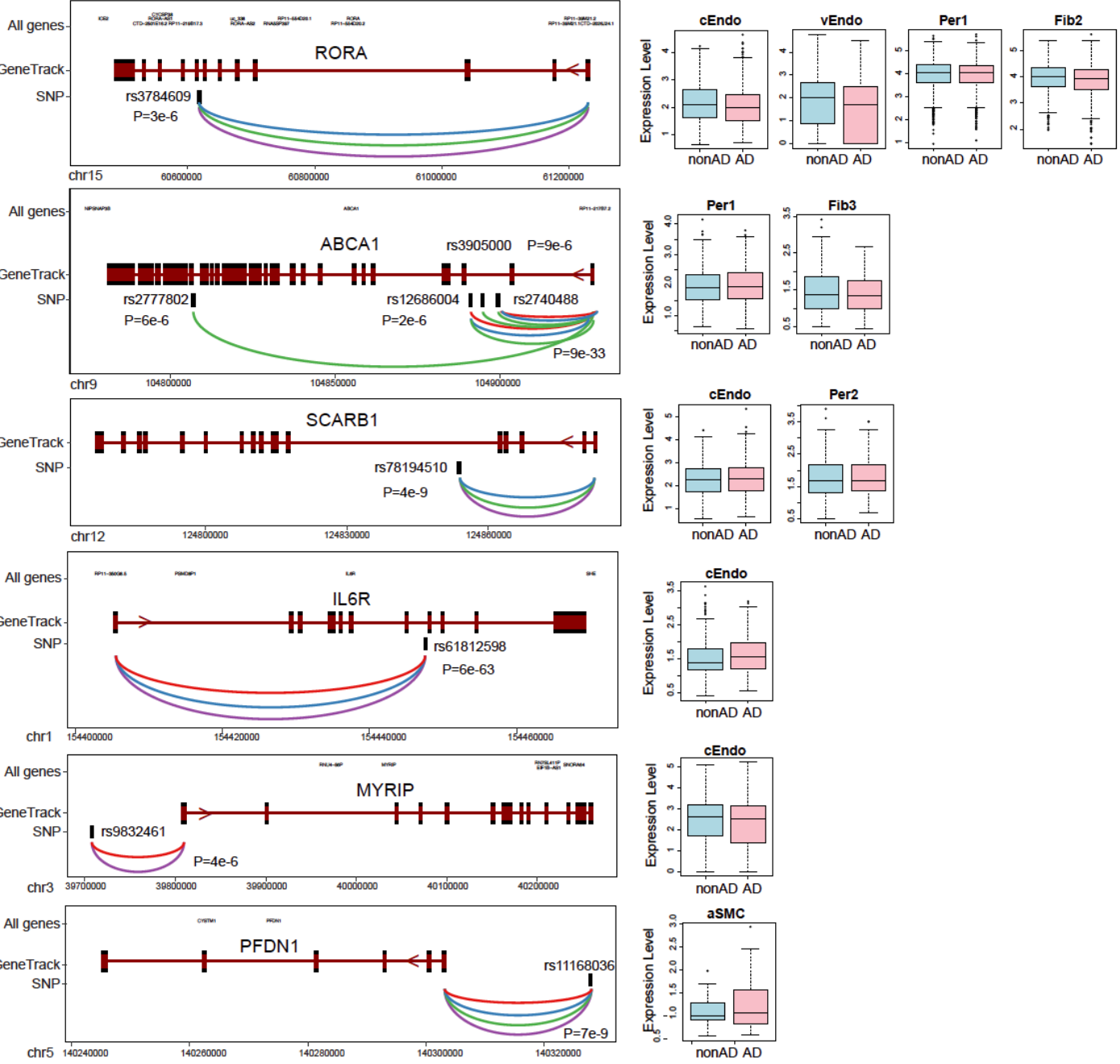

Linking type

- EpiMap correlation _ sc-eQTL

- tissue-eQTL $\quad-\mathrm{Hi}-\mathrm{C}$ loop

Extended Data Figure 5. Differentially expressed vascular genes association with AD genetics. a. Proposed three types of regulatory mechanisms to interpret the association between adDEGs and AD genetic variants: (1) Directly (cis) regulate adDEGs; (2) Indirect (trans) regulates adDEGs; (3) Indirect (ligand-receptor signaling) regulates adDEGs. b-g. Examples of adDEGs directly regulated by AD-associated variants through linking (eQTLs, Hi-C, promoter-enhancer correlation) along with the expression changes in vascular cell types. $\mathbf{h}$. The number of targets regulated by the three regulatory mechanisms. 
a

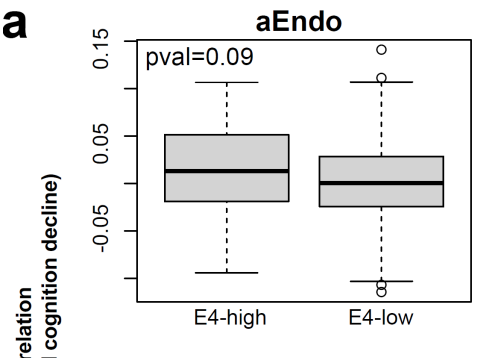

Fib3

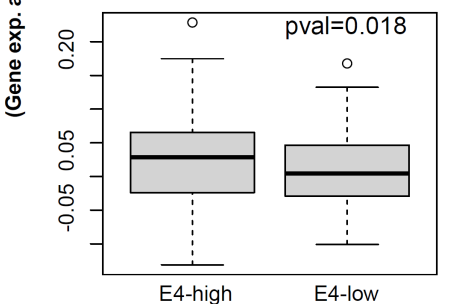

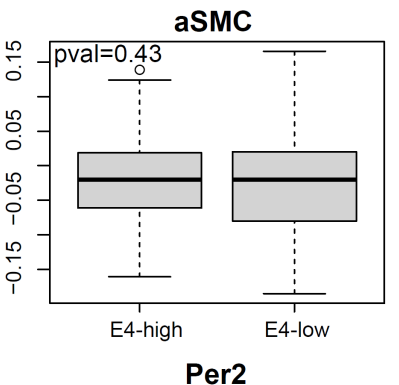

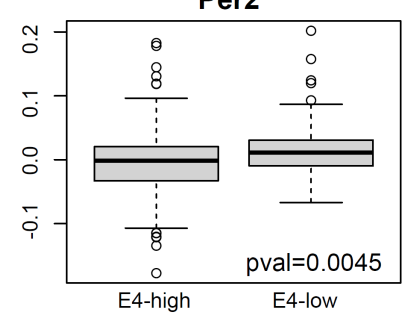

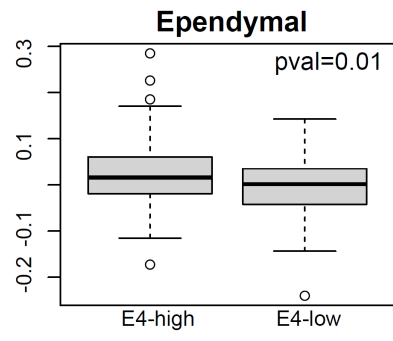

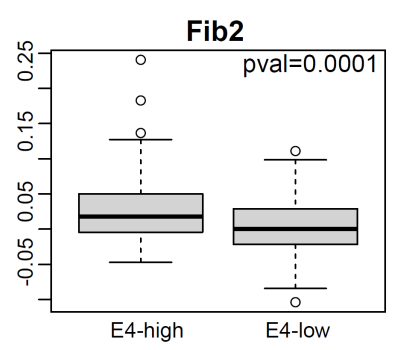

vSMC

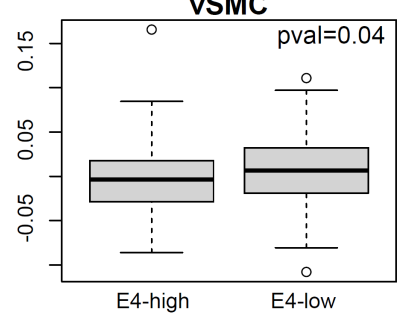

Extended Data Figure 6. APOE genotype, BBB dysfunction and cognitive decline. a. The comparison of apoeDEGs correlation with cognitive decline in each cell type. 


\section{Supplementary Tables}

Supplementary Table 1. Metadata for ROSMAP samples

Supplementary Table 2. Marker genes for cell type and subtypes

Supplementary Table 3. Functional enrichment for cell type markers

Supplementary Table 4. brain region brDEGs

Supplementary Table 5. Functional enrichment for brDEGs

Supplementary Table 6. AD adDEGs

Supplementary Table 7. Functional enrichment for AD adDEGs

Supplementary Table 8. Predicted regulators and their targets

Supplementary Table 9. Predicted cell-cell interactions

Supplementary Table 10. 125 GWAS genes and their variants, linking evidence, and functional enrichment

Supplementary Table 11. GWAS TFs, targets and functions

Supplementary Table 12. GWAS ligands, $\mathrm{CCl}$ and their targets

Supplementary Table 13. Summary of AD GWAS-associated vascular adDEGs

Supplementary Table 14. apoeDEGs between APOE3 and APOE4

Supplementary Table 15. Functional enrichment for apoeDEGs between APOE3 and APOE4

Supplementary Table 16. APOE genotype dependent cognitive-decline-associated genes

Supplementary Table 17. Functional enrichment for APOE genotype dependent cognitive-decline-associated genes 